\title{
The curious case of II Lup: a complex morphology revealed with SAM/NACO and ALMA
}

DOI:

10.1093/mnras/sty1903

\section{Document Version}

Accepted author manuscript

Link to publication record in Manchester Research Explorer

\section{Citation for published version (APA):}

Lykou, F., Zijlstra, A. A., Kluska, J., Lagadec, E., Tuthill, P. G., Avison, A., Norris, B. R. M., \& Parker, Q. A. (2018). The curious case of II Lup: a complex morphology revealed with SAM/NACO and ALMA. Monthly Notices of the Royal Astronomical Society, 480(11). https://doi.org/10.1093/mnras/sty1903

\section{Published in:}

Monthly Notices of the Royal Astronomical Society

\section{Citing this paper}

Please note that where the full-text provided on Manchester Research Explorer is the Author Accepted Manuscript or Proof version this may differ from the final Published version. If citing, it is advised that you check and use the publisher's definitive version.

\section{General rights}

Copyright and moral rights for the publications made accessible in the Research Explorer are retained by the authors and/or other copyright owners and it is a condition of accessing publications that users recognise and abide by the legal requirements associated with these rights.

\section{Takedown policy}

If you believe that this document breaches copyright please refer to the University of Manchester's Takedown Procedures [http://man.ac.uk/04Y6Bo] or contact uml.scholarlycommunications@manchester.ac.uk providing relevant details, so we can investigate your claim.

\section{OPEN ACCESS}




\title{
The curious case of II Lup: a complex morphology revealed with SAM/NACO and ALMA
}

\author{
Foteini Lykou, ${ }^{1,2,3 \star} \dagger$ A.A. Zijlstra, ${ }^{2,4} \ddagger$ J. Kluska, ${ }^{5,6}$ E. Lagadec, ${ }^{7}$ P.G. Tuthill, ${ }^{8}$ \\ A. Avison, ${ }^{4}$ B.R.M. Norris, ${ }^{8}$ Q.A. Parker ${ }^{1,2}$ \\ ${ }^{1}$ Department of Physics, The University of Hong Kong, Chong Yuet Ming Physics Building, Pokfulam Road, Hong Kong \\ ${ }^{2}$ Laboratory for Space Research, The University of Hong Kong, Block A, Cyberport 4, 100 Cyberport Rd, Cyberport, Hong Kong \\ ${ }^{3}$ Institute for Astrophysics, University of Vienna, Türkenschanzstrasse 17, A-1180 Vienna, Austria \\ ${ }^{4}$ Jodrell Bank Centre for Astrophysics, The University of Manchester, Oxford Road, M13 9PL, Manchester, UK \\ ${ }^{5}$ University of Exeter, School of Physics, Stocker Road, Exeter, EX4 4QL, UK \\ ${ }^{6}$ Institute for Astronomy, KU Leuven, Celestijnenlaan 200D B2401, 3001 Leuven, Belgium \\ ${ }^{7}$ Laboratoire Lagrange (UMR 7293), CNRS, Observatoire de la Cote d'Azur, Bd. de l'Observatoire, 06304 Nice Cedex 4, France \\ ${ }^{8}$ Sydney Institute for Astronomy, School of Physics, The University of Sydney, NSW 2006, Australia
}

Accepted XXX. Received YYY; in original form ZZZ

\begin{abstract}
We present the first-ever images of the circumstellar environment of the carbon-rich AGB star II Lup in the infrared and sub-mm, and the discovery of the envelope's nonspherical morphology with the use of high-angular resolution imaging techniques with the sparse aperture masking mode on NACO/VLT (that enables diffraction limited resolution from a single telescope) and with ALMA. We have successfully recovered images in $K s(2.18 \mu \mathrm{m}), L^{\prime}(3.80 \mu \mathrm{m})$ and $M^{\prime}(4.78 \mu \mathrm{m})$, that revealed the non-spherical morphology of the circumstellar envelope around II Lup. The stellar surface of the AGB star is unresolved (i.e. $\leq 30$ mas in $K s$ ) however the detected structure extends up to 110 mas from the star in all filters. Clumps have been found in the $K s$ maps, while at lower emission levels a hook-like structure appears to extend counter-clockwise from the South. At larger spatial scales, the circumstellar envelope extends up to approximately $23^{\prime \prime}$, while its shape suggests a spiral at four different molecules, namely $\mathrm{CO}, \mathrm{SiO}, \mathrm{CS}$ and $\mathrm{HC}_{3} \mathrm{~N}$, with an average arm spacing of $1.7^{\prime \prime}$ which would imply an orbital period of 128 years for a distance of 590pc.
\end{abstract}

Key words: instrumentation: high angular resolution - methods: observational techniques: interferometric - stars: AGB and post-AGB - stars: imaging - stars: individual: II Lup

\section{INTRODUCTION}

In the last decade significant steps have been made in understanding stellar mass loss during the asymptotic giant branch (AGB) stage of low- to intermediatemass stars $\left(M_{\text {init }}=0.8-8 \mathrm{M}_{\odot}\right)$. Although the precise mechanisms for mass loss remain contentious, some interplay between pulsation, dust formation and radiative driving is highly likely. This has been demonstrated, for carbon stars, by dynamic models of Höfner et al. (2003), Nowotny et al. (2005), Mattsson et al. (2010),

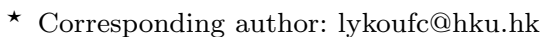

$\dagger$ Based on observations made with ESO Telescopes at the La Silla Paranal Observatory under programme IDs 085.D-0356 and 60.A-9637.

$\ddagger$ Hung Hing Ying Distinguished Visiting Professor
}

Nowotny et al. (2011), Liljegren et al. (2016), and their applications to high-angular interferometric observations in, e.g., Wittkowski et al. (2017), Rau et al. (2017), Ohnaka et al. (2015), Rau et al. (2015), Ohnaka et al. (2007). For oxygen-rich stars, the interplay has been demonstrated by Bladh et al. (2015), and the interferometric observations by Wittkowski et al. (2018), Karovicova et al. (2013) and Ohnaka et al. (2012).

For the case of carbon-rich stars in particular $(M \leq$ $\left.4 \mathrm{M}_{\odot}\right)$, it has been suggested that these stars produce high mass-loss yields, and are therefore important players in the chemical enrichment of the interstellar medium in the solar neighbourhood (e.g., Mattsson 2010; Schröder \& Sedlmayr 2001). However, such predictions are also dependent on the metallicity and the model in use (e.g., Karakas \& Lugaro 2016). Nowotny et al. (2013, and references therein) have shown that increasing mass-loss rates in carbon-rich AGB 
Table 1. II Lup astrometry.

\begin{tabular}{|c|c|c|c|}
\hline Survey & RA (J2000) & Dec (J2000) & Epoch \\
\hline USNO-B1.0 & 152305.154 & -512558.65 & 1986.7 \\
\hline USNO-B1.0 & 152305.214 & -512559.19 & 1987.4 \\
\hline PPMXL & 152305.091 & -512558.79 & 1989.91 \\
\hline PPMXL & 152305.106 & -512558.94 & 1991.43 \\
\hline GSC2.2 & 152305.077 & -512558.58 & 1997.288 \\
\hline GSC 2.3 & 152305.084 & -512558.82 & 1992.56 \\
\hline 2MASS & 152305.075 & -512558.73 & 1999.441 \\
\hline DENIS & 152305.072 & -512558.84 & 1998.458 \\
\hline SHS & 152305.083 & -512558.89 & 1999.505 \\
\hline $\mathrm{HST}^{a}$ & 152304.999 & -512558.29 & 2004.611 \\
\hline Gaia DR2 & 152305.0744 & -512558.881 & 2015.5 \\
\hline
\end{tabular}

${ }^{a}$ Position derived from SExtractor photometry in the Hubble Legacy Archive.

stars will result in higher circumstellar reddening, and therefore these stars will appear more obscured (see also Liljegren et al. 2016) .

On the subject of what shapes the stellar ejecta, the two most-favoured mechanisms are magnetic fields and binarity, although of course these two are not mutually exclusive and hybrid models may also be favoured. If the morphology is governed by binarity, the oxygen-rich and carbon-rich stars should show the same range of morphologies in their circumstellar structures, but confirming this will require a large sample of sources.

The shape of the stellar ejecta beyond the AGB phase, as witnessed in the majority of imaging surveys (e.g., Castro-Carrizo et al. 2010; Lagadec et al. 2011), often departs from spherical symmetry (hereafter, asymmetry). This is more evident in later evolutionary stages, such as the planetary nebula phase where only about $20 \%$ of planetary nebulae have been found to be spherically symmetric (Parker et al. 2006). With the advancement of observational techniques, it is now possible to detect such morphological changes even within two stellar radii from the surface of AGB stars.

As part of an on-going effort in detecting asymmetries in the ejecta of AGB stars (e.g., Lykou et al. 2015; Lagadec et al. 2011; Tuthill et al. 2000a,b), we present here our investigation of one such star, II Lup (Section 2). This work is based on single-dish and on interferometric observations (Section 3) and the results are compared with historical findings from speckle imaging (Section 4). We present the first-ever images of II Lup in the near-infrared (Section 5) and the sub-mm (Section 7.2) wavelengths. We derive the physical parameters of II Lup in Section 6, discuss our imaging results in Section 7, and present our final conclusions in Section 8 .

\section{THE CASE OF II LUP}

The star II Lup was known as the third brightest AGB star in the southern hemisphere at $12 \mu \mathrm{m}$ when observed by IRAS (Meadows et al. 1987; Nyman et al. 1993). It was first mentioned as WO48 in the survey of Westerlund \& Olander (1978) and misclassified as an S-star. It was later observed by the IRAS space telescope (IRAS 15194-5115; Neugebauer et al. 1984) and si-

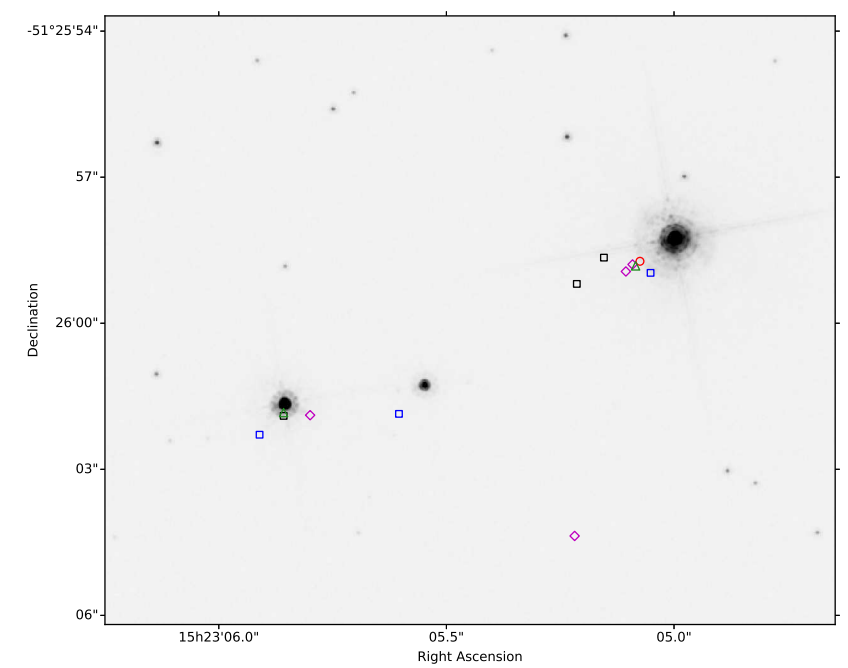

Figure 1. A field image of II Lup taken with the Hubble Space Telescope's ACS/HRC camera and the F606W filter. II Lup is the brightest star in the field, and the region is overlayed with the pointing of several surveys, namely Gaia/DR2 (blue boxes), 2MASS (red circle), GSC2.3 (green triangles), PPMXL (magenta diamonds) and USNO-B.1 (black boxes).

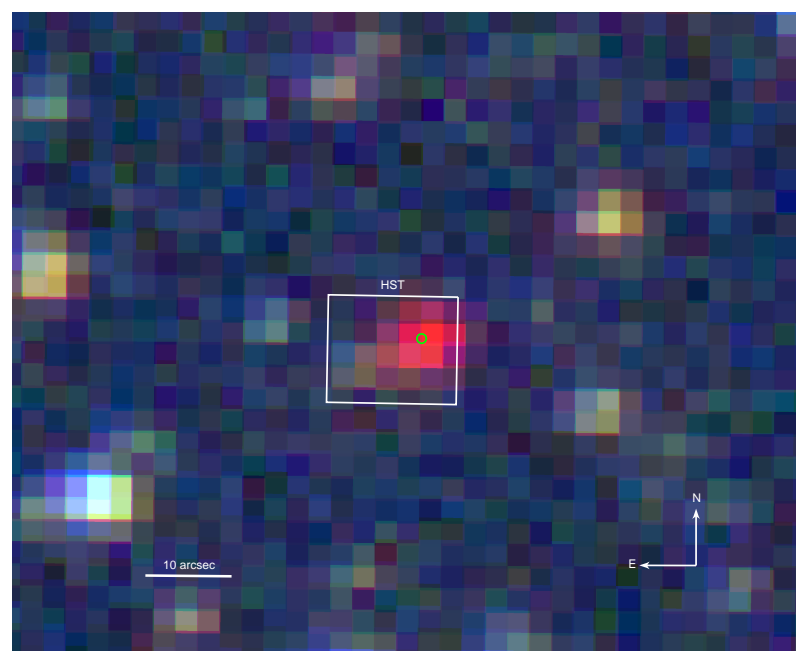

Figure 2. A three-colour image of the field around II Lup from the AAVSO Photometric All-Sky Survey (APASS) in 2011. The colours blue, green and red stand for the filters Johnson $B, V$ and Sloan $i^{\prime}$, respectively. The entire field-of-view shown in Fig. 1 is indicated by a white box for comparison. II Lup was not detected in filters $B$ and $V$ (probably fainter than $16.5 \mathrm{mag}$ in $V$ ). The oblate morphology of the source within the box is in part due to the poor angular resolution and focus of the APASS survey, and due to the nearby, eastern field stars in Fig. 1 which are also bright in $i^{\prime}$. The position of II Lup in the Gaia system is marked with a green circle.

multaneously identified in the Valinhos infrared survey (Epchtein et al. 1987). Meadows et al. (1987) characterised II Lup as a C-rich star based on the carbonaceous species found in its near-infrared spectrum ( $3.1 \mu \mathrm{m}$ feature). 
Table 2. Modelled stellar and envelope radii from the literature vs. the angular size for an adopted distance of $590 \mathrm{pc}$.

\begin{tabular}{cccc}
\hline$R_{*}[\mathrm{AU}]$ & $R_{\text {env }}[\mathrm{AU}]$ & Reference & $\theta_{\text {env }}[\mathrm{mas}]$ \\
\hline 1.86 & 10.02 & $(1)$ & 16.99 \\
3.00 & 12.03 & $(2)$ & 20.38 \\
2.54 & 7.19 & $(3)$ & 12.19 \\
2.66 & 27.20 & $(4)$ & 46.10 \\
2.52 & 10.02 & $(5)$ & 16.99 \\
2.32 & 16.48 & $(6)$ & 27.93 \\
3.54 & 21.24 & $(7)$ & 36.00 \\
2.52 & 14.70 & $(8)$ & 24.92 \\
\hline
\end{tabular}

References: (1) Ramstedt \& Olofsson (2014);

(2) Ryde et al. (1999); (3) De Beck et al. (2010);

(4) Dehaes et al. (2007); (5) Schöier et al. (2007);

(6) Groenewegen et al. (1998); (7) Lopez et al. (1993);

(8) Danilovich et al. (2015)

\subsection{On the astrometry and distance.}

Although a bright infrared source, there is no optical identification published. II Lup is not included in the Tycho and Hipparcos catalogues (ESA 1997), but it is included in Gaia DR2 (Gaia Collaboration et al. 2016, 2018). However, the current Gaia parallax ( $\pi=0.52 \pm 0.28$ mas $)$ is not reliable, since parallax estimation is problematic for extremely red and variable sources such as II Lup (Mowlavi et al. 2018, and references therein). Figure 1 shows the field near II Lup as viewed by the Hubble Space Telescope (HST) at $0.6 \mu \mathrm{m}$ (ACS/HRC filter F606W; prog.ID 10185). We extracted astrometric information on II Lup using the Aladin ${ }^{1}$ tool (Bonnarel et al. 2000).

Comparing the coordinates given for the field stars and II Lup from five major catalogues, namely Gaia (Gaia Collaboration et al. 2016, 2018), 2MASS (Cutri et al. 2003), PPMXL (Roeser et al. 2010), GSC2.3 and USNO-B.1 (Monet et al. 2003), to the HST image in Figure 1 clearly shows the mismatch between the various astrometric solutions (cf. Table 1). The difference in coordinates of the two bright field stars South-East of II Lup between the HST and the Gaia systems are less than $1^{\prime \prime}$, which is similar to the known variations, depending on the field, of the astrometric uncertainty of the Guide Star Catalogue. The uncertainties both in the coordinates and proper motions of the remaining abovementioned catalogues, indicates that they have all detected the same star: II Lup. Thus, there is an optical counterpart to this deeply embedded star.

Taking into account the inefficacy of the current Gaia algorithm in determining accurate parallaxes for variable stars like II Lup, if we consider the $3 \sigma$ limit of the Gaia DR2 parallax, we do not expect II Lup to be closer than 500 pc. We have adopted the distance of 590 pc proposed by Groenewegen et al. (2002a) and Menzies et al. (2006). This distance was estimated from the period-luminosity relation for carbon-rich Miras of Whitelock et al. (2006).
Table 3. Photometry of II Lup.

\begin{tabular}{|c|c|c|c|}
\hline Band & $m$ & & Reference \\
\hline$B$ & 17.85 & & NOMAD \\
\hline$V$ & 16.66 & & NOMAD \\
\hline$R$ & 14.51 & & NOMAD \\
\hline$I$ & 10.54 & & DENIS \\
\hline$J$ & 7.28 & & $(1,2)$ \\
\hline$H$ & 4.58 & & $(1,2)$ \\
\hline$K$ & 2.35 & & $(1,2)$ \\
\hline$L$ & -0.56 & & (1) \\
\hline$M$ & -1.49 & & (1) \\
\hline$\lambda[\mu \mathrm{m}]$ & $F[\mathrm{Jy}]$ & $F_{\text {err }}[\mathrm{Jy}]$ & Reference \\
\hline 3.08 & 459 & 12 & ISO \\
\hline 3.35 & 37.5 & 0.3 & WISE \\
\hline 3.35 & 1255.2 & - & AllWISE \\
\hline 3.5 & 203.3 & 30.5 & COBE \\
\hline 4.29 & 371.4 & 31 & MSX \\
\hline 4.6 & 83.4 & 0.3 & WISE \\
\hline 4.6 & 78.7 & - & AllWISE \\
\hline 4.9 & 500.6 & 36.4 & COBE \\
\hline 6.3 & 1000 & 100 & ISO (3) \\
\hline 8.28 & 193.1 & 7.9 & MSX \\
\hline 8.8 & 860 & 86 & ISO (4) \\
\hline 9.8 & 852 & 85 & ISO (4) \\
\hline 11.6 & 427.6 & 5 & WISE \\
\hline 11.6 & 574.2 & - & AllWISE \\
\hline 11.7 & 860 & 86 & ISO (4) \\
\hline 12 & 1310 & 104.8 & IRAS \\
\hline 12 & 818.8 & 55.5 & $\mathrm{COBE}$ \\
\hline 12.13 & 861.8 & 43.1 & MSX \\
\hline 12.5 & 681 & 68 & ISO (4) \\
\hline 14.65 & 630.7 & 38.4 & MSX \\
\hline 18 & 526.5 & 38.1 & AKARI \\
\hline 21.24 & 488.3 & 29.3 & MSX \\
\hline 22.1 & 676 & - & WISE \\
\hline 22.1 & 248 & 0.45 & AllWISE \\
\hline 25 & 553.7 & 33.2 & IRAS \\
\hline 25 & 606.8 & 51.5 & $\mathrm{COBE}$ \\
\hline 60 & 225.7 & 101 & $\mathrm{COBE}$ \\
\hline 60 & 129.9 & 12.99 & IRAS \\
\hline 65 & 107.9 & 8.35 & AKARI \\
\hline 70 & 106 & 10.6 & Herschel (5) \\
\hline 90 & 62.9 & 1.67 & AKARI \\
\hline 100 & 52.57 & 6.3 & IRAS \\
\hline 140 & 16.14 & 1.17 & AKARI \\
\hline 160 & 14.97 & 1.03 & AKARI \\
\hline 160 & 22.9 & 3.4 & Herschel (5) \\
\hline 1200 & 0.3106 & 0.019 & $(6)$ \\
\hline
\end{tabular}

References: (1) Le Bertre (1992); (2) Whitelock et al. (2006);

(3) Neufeld et al. (2011); (4) Guandalini et al. (2006);

(5) Groenewegen et al. (2011); (6) Dehaes et al. (2007)

\subsection{Photometry}

There is no available time-series photometry for this star in the visual ${ }^{2}$. The only available photometry in $V$ from the literature is found in the work of Meadows et al. (1987), where the authors give an upper limit of $V>21$, and in the NOMAD catalogue $(B=17.85$ and $V=16.66$; Zacharias et al.

2 No data found in the archives of AAVSO, ASAS, OGLE or Hipparcos. 

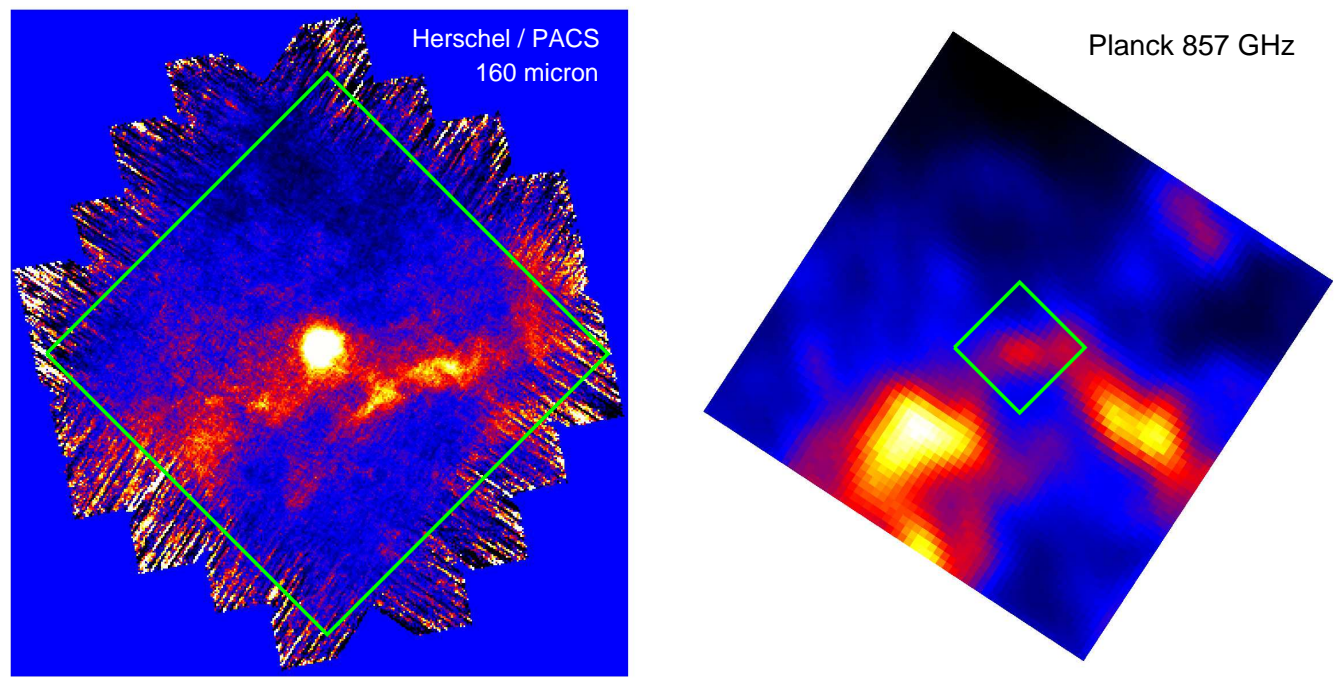

Figure 3. Far-infrared region maps of II Lup from the Herschel $(160 \mu \mathrm{m}$; left) and Planck (857GHz; right) space telescopes. Some filaments of the background Lupus cloud are clearly visible in the Planck map (size $1^{\circ} \times 1^{\circ}$ ) with brighter clumps seen more than $0.5^{\circ}$ South-East and South-West from II Lup (centre). The green boxes are $0.2^{\circ} \times 0.2^{\circ}$ in both panels. North is up and East is left.

2004). The AAVSO Photometric Sky Survey (APASS) did not detect the star in either $B$ or $V$, suggesting that the star was fainter than $16.5 \mathrm{mag}$ in $V$ in 2011 (see also Fig. 2). II Lup is included in the Catalog for Optically Variable Sources of the Optical Monitoring Camera (OMC) of the INTEGRAL space telescope ${ }^{3}$ (Alfonso-Garzón et al. 2012), which estimated $15.5 \lesssim V \lesssim 14.4$ over a period of several years (2003-2016). However OMC has a pixel size of $17.6^{\prime \prime}$ and it is mentioned that the results were of poor photometric quality. We note that the stellar field of Fig. 1 falls within one OMC pixel, which may explain the poor photometric quality ${ }^{4}$.

The reported colour for II Lup in the Gaia system is $G_{\mathrm{BP}}-G_{\mathrm{RP}}=6.26$ and $G=14.262$. The given magnitudes for II Lup in the Hubble Legacy Archive is $[F 606 W]=15.74$ and $[F 814 W]=12.26$, which is clearly the brightest star in the field in Fig. 1. In Table 3, we have compiled the available photometry from the literature ${ }^{5}$.

\subsection{On the circumstellar environment in the infrared}

The chemical composition of II Lup has been extensively studied through terrestrial single-dish observations in the far-infrared and radio regimes. Various radiative transfer models, that assumed a spherical geometry for the circumstellar envelope, have been applied to these observations (Nyman et al. 1993; Ryde et al. 1999; Groenewegen et al.

3 sdc.cab.inta-csic.es/omc/index.jsp

4 Bad centroid or centroid too far from the source's coordinates.

5 Only the mean $R, I, J, H, K, L, M$ photometry is tabulated for the sake of clarity, and only known flux uncertainties are listed.
1999; Schöier et al. 2002; Groenewegen et al. 2002b; Dehaes et al. 2007; De Beck et al. 2010; Neufeld et al. 2011; Smith et al. 2012; Ramstedt \& Olofsson 2014). The results of the modelling vary depending on the adopted distances and luminosities, as well as the radiative transfer model in use. However there is an overall agreement on the gas mass loss which is $5-20 \times 10^{-6} \mathrm{M}_{\odot} \mathrm{yr}^{-1}$. On the other hand, values for the dust mass loss vary from $1-8 \times 10^{-8} \mathrm{M}_{\odot}$ $\mathrm{yr}^{-1}$ (Groenewegen et al. 1998, 2002b; Smith et al. 2012), again depending on the radiative transfer model applied on the spectral energy distribution. The modelled stellar radii and inner radii of the envelope $\left(R_{\text {env }}\right)$ derived from the literature are tabulated in Table 2 for reference. We have converted the inner radii $R_{\text {env }}$ into angular sizes $\left(\theta_{\text {env }}\right)$ for an adopted distance of $590 \mathrm{pc}$.

We searched for any available image of II Lup in all (space and ground) telescope data archives in the infrared regime. Due to the star's extreme brightness in the nearand mid-infrared, the images found in the DENIS, 2MASS, WISE, MSX and IRAS archives, are either saturated or indicate that II Lup was unresolved. In the far-infrared, the circumstellar envelope of II Lup is resolved in the AKARI, Herschel and Planck images beyond $65 \mu \mathrm{m}$. These images also reveal background emission from the Lupus interstellar cloud (Fig. 3). II Lup is much brighter than the background emission in all images. This may be further justified by the lower extinction calculated by Menzies et al. (2006) (0.44 $\left.\leq A_{V} \leq 0.48\right)$ and Groenewegen et al. (1998) $\left(A_{V}=0.8\right)$ compared to that of Schlafly \& Finkbeiner (2011) $\left(A_{V}=2.38\right)$. The last is based on an analysis of the Sloan Digital Sky Survey, as well as far-infrared temperature maps from the DIRBE/COBE and IRAS surveys. The size of II Lup's circumstellar cloud as determined from the $70 \mu \mathrm{m}$ image is approximately $40^{\prime \prime}$, however it bears no distinctive 


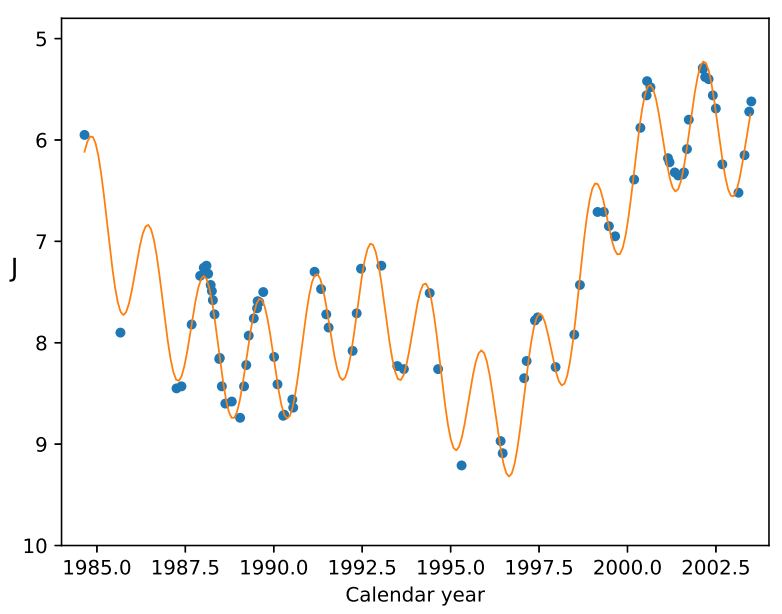

Figure 4. PERIODO4 fit to the $J$ band light curve. Photometry extracted from: Epchtein et al. (1987); Le Bertre (1992); Feast et al. (2003); Whitelock et al. (2006).

morphological features other than a round shape (Cox et al. 2012; Groenewegen et al. 2011).

\subsection{On the peculiar secondary period}

II Lup has been monitored in the near-infrared $(J, H$, $K, L$ and $M$ ) over the course of approximately 20 years (Epchtein et al. 1987; Le Bertre 1992; Feast et al. 2003; Whitelock et al. 2006). Feast et al. (2003) presented the first comprehensive study of II Lup's photometric variability. From their analysis, the authors derived a primary period of 575 days and a secondary period of approximately 19 years (6900 days); with the longer period proposed to result from an obscuration event. Such events have been observed before in other AGB stars and appear to be relatively common. Prominent examples are the stars V Hya (Knapp et al. 1999), R Lep, R For, EV Eri (Whitelock et al. 2006), L2 Pup (Bedding et al. 2002), and M2-29 (Hajduk et al. 2008).

Feast et al. (2003) argue that the extreme variability at the shorter wavelengths ( $J$ to $K$ ) is due to scattering by dust, which was suggested by Lopez et al. (1993) as well. Feast et al. suggested the presence of dusty puffs around II Lup. From its IRAS colours ([12] $-[25]=-0.921$, and $[25]-[60]=-1.476)$ and its intrinsic variability in the nearinfrared, II Lup is somewhat similar to a class of variable carbon-rich stars known as $\mathrm{R}$ CrB stars. The principle star of this class, $\mathrm{R} \mathrm{CrB}$, has similar IRAS colours $(-0.924$ and -1.57 , respectively) and is also known to have ejected large dust puffs in the past (Jeffers et al. 2012; Clayton et al. 2011). However, the variability of II Lup is not as rapid as that of $\mathrm{R} \mathrm{CrB}$, where typical primary pulsation periods of 40 days are found in the visual light curves (Crause et al. 2007).

According to Whitelock et al. (2006) obscuration events, such as the one proposed for the case of II Lup, are found in one-third of carbon-rich Miras in their sample. According to Olivier \& Wood (2003), such long secondary periods can be explained either by an expulsion of dusty clumps (such as those observed in R CrB stars: Jeffers et al. 2012; Clayton et al. 2011) or by the presence of a companion. Olivier \& Wood (2003) and Wood \& Nicholls (2009) have confirmed the binary nature of many stars with long secondary periods in the LMC, while at least two cases are known within our Galaxy: e.g., L2 Pup (Kervella et al. 2016) and V Hya (Knapp et al. 1999).

Additional time-series infrared photometry was found in the DIRBE/COBE (Price et al. 2010) and WISE survey databases. Price et al. (2010) suggest a period of 632 days from the $L$ and $M$ DIRBE time-series. This period is significantly longer than the derivations of Feast et al. (2003) and Menzies et al. (2006). We note that WISE photometry is available for only two epochs and may suffer from saturation in all bands.

We have re-analysed all available infrared timeseries photometry from the literature with PERIOD04 (Lenz \& Breger 2004), a software commonly used in asteroseismology. From this new analysis and the inclusion of the DIRBE/COBE data, the primary period of 575 days derived by Feast et al. is confirmed (this work: $574 \pm 2$ days), as well as the secondary period (this work: $19.7 \pm 0.2$ years). Variations were found in the secondary period in $H$ and $K$ bands ( $\simeq 25$ years), however Feast et al. did not report any secondary period calculations in those bands. PERIOD04 provided a good fit to the $J$ band photometry (Fig. 4), while the distinctive brightening after 1996, as mentioned by Feast et al. (their Fig.1), is also satisfied by the synthetic light curve of PERIOD04. A variation in the calculation of the secondary period was expected. This is caused mainly by the uneven sampling in the time series especially from 2003 until now. Below we will argue that the orbital period is much longer; the derived secondary period may therefore reflect the limited time coverage rather than the true value.

\section{OBSERVATIONS AND DATA REDUCTION}

\section{$3.1 \quad$ NACO}

II Lup was observed with the NACO instrument on 29/30th June and 1st July 2010 (Prog.ID: 085.D-0356) at the UT4 telescope of VLT (Lenzen et al. 2003; Rousset et al. 2003). We used the Sparse Aperture Masking mode (SAM; Tuthill et al. 2010) where a mask is introduced in the pupil plane and in essence converts the full aperture into a Fizeau interferometer (Tuthill et al. 2000a).

One can retrieve information, extending beyond the diffraction-limit of the telescope, from the interference pattern created by the multiple apertures, by measuring the amplitude and phase of the fringes. A large number of apertures allows good Fourier coverage and a high fraction of the phase information recovered by way of the closure phases. An illustration of a typical example of the interference pattern created by SAM is given in Figure 5 .

We have used the 9-hole mask where each hole corresponds to an effective aperture of 0.92 metres. This mask provides 36 baselines with baseline length ranging from 1.3 to 6.9 metres (Fig. 5). Three broadband filters were used, $K s(2.18 \mu \mathrm{m}), L^{\prime}(3.8 \mu \mathrm{m})$ and $M^{\prime}(4.78 \mu \mathrm{m})$, where the angular resolution of the synthesized beam is $32.5,56.8$ and 71.4 mas, respectively. During the first night the seeing fluctuated between 1 and 2.5 arcsec, where the observations in 

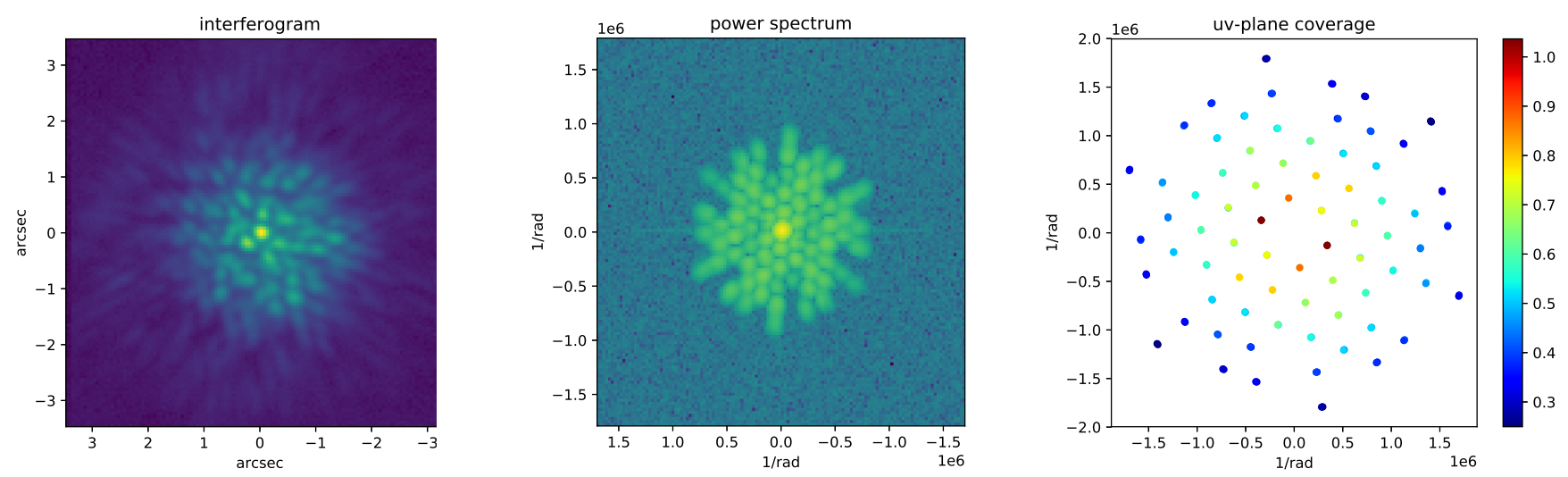

Figure 5. Left: An example of the interference pattern created by the 36 apertures of the 9-hole mask. The first Airy ring is visible (online version of this figure) suggesting that the object was resolved. North is up and East is on the left. Centre: the power spectrum of the given interferogram. Each spot corresponds to the sampled visibility and its complex conjugate. Right: the $u v$-plane coverage with the 9-hole mask for II Lup. The points are colour-coded with increasing values of squared visibility amplitude.

$L^{\prime}$ and $M^{\prime}$ suffered from poor seeing. The $K s$ band observations were carried out during better conditions on the second night. NACO has four different focal cameras and we used the S27 for the $K s$ band and the L27 camera for the other two broadband filters (for both cases the pixel size is 27.1 mas). We chose a window subframe of $256 \times 256$ pixels.

Two hours were dedicated to the observations of II Lup and its calibrators. The calibrators measure the telescope transfer function, i.e. the visibility amplitudes of a point source. Thus, the data are calibrated using observations of an unresolved star. The telescope transfer function depends only on the mask and its orientation on the pupil, which remains stable since the pupil-tracking mode was used. In this mode the pupil is kept constant while the sky rotates and the field rotation is later corrected during data reduction. The resolution and the transfer function of the telescope are not dependent on the seeing, but the signal-to-noise is reduced at poorer seeing.

We followed the standard data reduction process for SAM data using a pipeline developed at the University of Sydney (Tuthill et al. 2000a; Lacour et al. 2011). For each data cube, the individual speckle frames were flat-fielded, dark, bias, and sky subtracted. In the next step, the frames were Fourier transformed, and the extracted power spectra and bispectra (i.e., the Fourier components) were calibrated. The $L^{\prime}$ and $M^{\prime}$ bands data were calibrated using calibrators from both the first and the second night of observations. No significant difference in the telescope transfer function calibration was found overall.

Sparse aperture masking does not provide images, but model images can be derived from the power spectra and the closure phases through image reconstruction methods. We have used two such methods (Sec. 5), MACIM (Ireland et al. 2006) and MiRA (Thiébaut 2008) which differ in the model used for the image reconstruction (see also Lykou et al. 2015; Monnier et al. 2014; Baron et al. 2012, for a comparison between the different methods).

\section{$3.2 \quad$ VISIR}

We obtained images of II Lup in the mid-infrared with the VISIR instrument on the VLT on 21 March $2016^{6}$ (Prog. ID: 60.A-9637). The observations were part of the scientific verification program for the instrument's upgrade. We have used the burst mode ${ }^{7}$ with a $0.045^{\prime \prime}$ pixel scale, the PAH_1 $\left(\lambda_{\mathrm{c}}=8.54 \mu \mathrm{m}, \Delta \lambda=0.42 \mu \mathrm{m}\right)$ and PAH_2 $\left(\lambda_{\mathrm{c}}=\right.$ $11.25 \mu \mathrm{m}, \Delta \lambda=0.59 \mu \mathrm{m})$ filters, and HD133550 as a PSF calibrator. The chopping and nodding sequence was used to allow for background subtraction and the final reconstruction of images with a shift-and-add technique, i.e. bad pixels were removed from each image and subsequently each image was shifted and added using a maximum of correlation algorithm. The total time on target was 32 minutes. A similar process was followed for the PSF calibrator observations.

The raw images indicated that II Lup is unresolved in both filters as indicated by the presence of an extended Airy ring pattern, while many images showed instrumental effects due to saturation ("bleeding"). Intensity cuts, bisecting the images north-south and east-west from the central pixel, showed the consecutive Airy rings around the core, while the core's flat plateaus suggest that these images suffer from saturation. We attempted to deconvolve the images using the maximum likelihood method and Lucy-Richardson deconvolution (Landsman 1993), however this did not improve the images. Therefore, we expect that the circumstellar envelope of II Lup in the mid-infrared is less than $0.25^{\prime \prime}$ in size. Due to the poor quality of the VISIR images, we opted to not present the raw data, as they are unreliable for any further analysis.

\section{$3.3 \quad$ ALMA}

The ALMA data, from project 2013.1.00070.S, were extracted from the ALMA archive and processed using the reduction scripts from the archive to re-create the fully calibrated measurement set. The ALMA observations were

$6 J D=2457468.366$ and $\phi=0.90$, cf. Sec. 4.1

7 The exposure time was $25 \mathrm{~ms}$ for PAH_1 and $27.8 \mathrm{~ms}$ for PAH_2. 

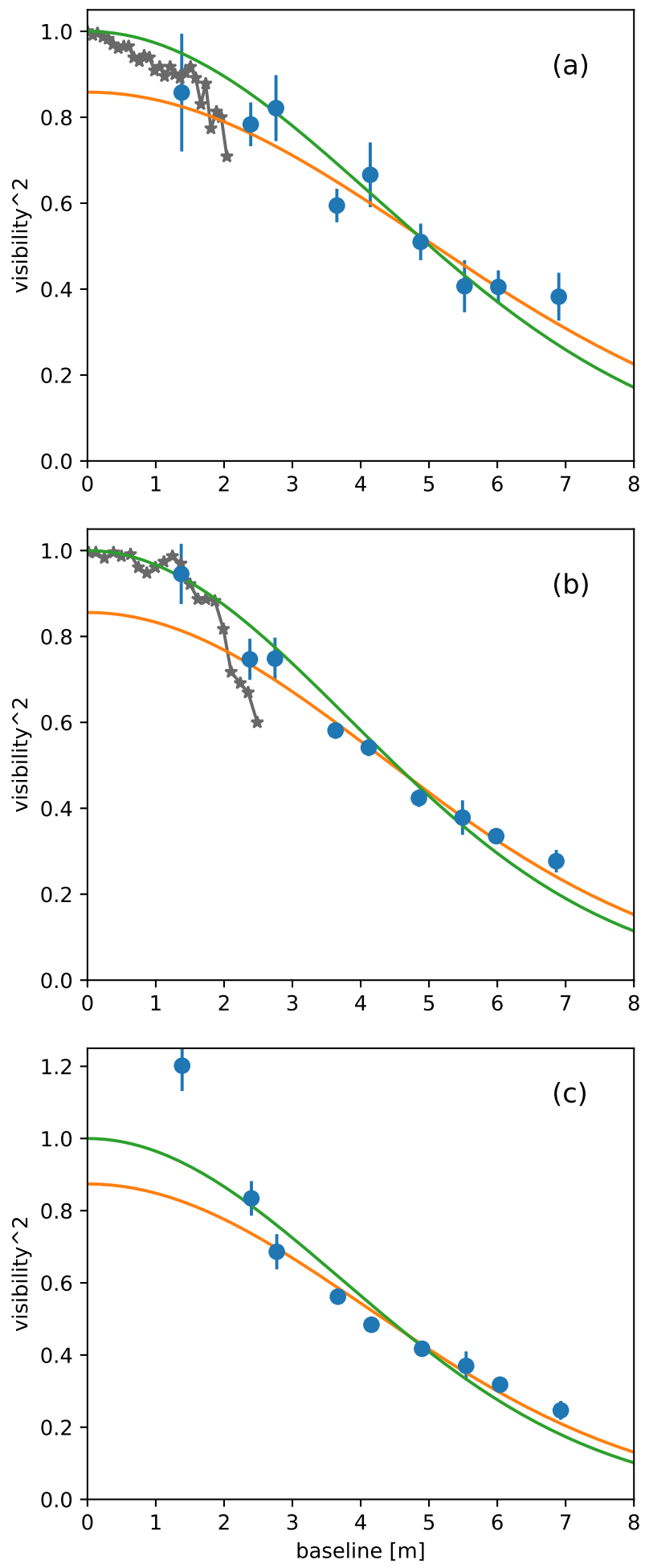

Figure 6. The azimuthally averaged SAM visibilities (blue circles) in $K s$ (a), $L^{\prime}$ (b) and $M^{\prime}$ (c). Simple centrosymmetric Gaussial distributions (green and orange lines) have been fitted to the SAM data. The measurements of Lopez et al. are shown for reference (gray stars, cf. Sec. 4.1).
Table 4. Equation 1 fitting results and envelope flux contribution.

\begin{tabular}{lc}
\hline filter & $\theta$ (mas) \\
\hline$K s$ & $34.4 \pm 2.2$ \\
$L^{\prime}$ & $69.2 \pm 1.5$ \\
$M^{\prime}$ & $90.3 \pm 2.2$ \\
\hline
\end{tabular}

conducted on 16th August 2015, in an array configuration containing 39 of the $12 \mathrm{~m}$-antennas with minimum and maximum baselines of $15.06-\mathrm{m}$ and $1574.37-\mathrm{m}$ respectively. This provides a maximum resolution of $0.42^{\prime \prime}$ at frequency $\sim 115.27 \mathrm{GHz}$ (to cover the ${ }^{12} \mathrm{CO} 1-0$ ) transition.

The calibrated data were imaged in CASA ver.5.1.1-5 (McMullin et al. 2007), with the task tclean, using a natural weighting of the visibilities and a uv-taper of $0.7^{\prime \prime}$ to taper the signal from the longest baselines. Visibilities from the baselines less than $30-\mathrm{m}$ were also removed during imaging as they contributed a large scale artefact to the final image. The final image created has an RMS noise of $5 \mathrm{mJy}$ in a single $2.54 \mathrm{~km} \mathrm{~s}^{-1}$ line-free channel and has a synthesised beam of $1.144^{\prime \prime} \times 0.821^{\prime \prime}$, elongated East-West.

\section{ANGULAR SIZES}

We have azimuthally averaged the SAM data (Fig. 6) and fitted geometric models to calculate the angular sizes of the dusty envelope at each wavelength (see also Lykou et al. 2015; Sanchez-Bermudez et al. 2016). Although this is an oversimplified approach in treating data that sampled different position angles, this method provides initial estimates about the size of the circumstellar envelope that may later be used as a priori parameters in the image reconstruction process.

At a first glance (Fig. 6), the shape of the visibility curve (blue circles) at high spatial frequencies in both $K s$ and $L^{\prime}$ suggests that the object may be resolved. We first fit a uniform disc model to the data. The fit was successful for the $K s$ data, but failed to fit the $L^{\prime}$ and $M^{\prime}$ data. As a second step, we fit a Gaussian distribution to the SAM data (orange line, Fig. 6), whose visibility function, $V(r)$, is expressed as:

$V(r)^{2}=\left[\frac{f_{\text {Gaussian }}}{f_{\text {Gaussian }}+f_{\text {background }}}\right] \exp \left(-3.57 \theta^{2} r^{2}\right)$

where $\theta$ is the angular diameter, $r$ is the spatial frequency, coefficient $f_{\text {Gaussian }}$ is the squared correlated flux at zero baseline, and $f_{\text {Gaussian }}+f_{\text {background }}$ is the total of the correlated and uncorrelated fluxes (the factorial in Eq. 1 represents the fractional flux of the envelope; see also similar examples by Sanchez-Bermudez et al. 2016). The orange curve in Figure 6 represents the fit derived from this scaled model. It is evident that the intercept of the fitted curve at zero baseline is not at $V^{2}=1$, which suggests that the extended flux is over-resolved. For comparison, we show a Gaussian distribution were the coefficient's ratio in Eq.1 was forced to unity and therefore $V^{2}=1$ at zero baseline (green line, 

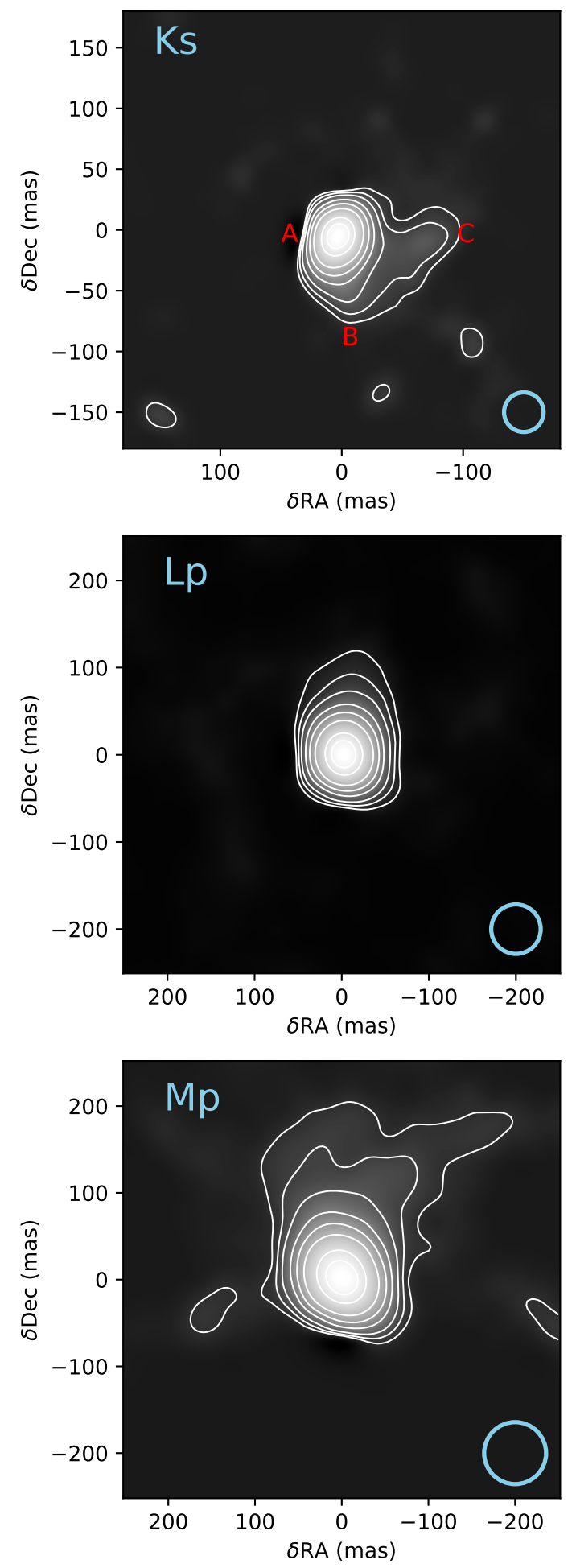

Figure 7. MACIM reconstructions for the $K s, L^{\prime}$ and $M^{\prime}$ data. Contour levels are at $0.5,1,2,5,10,20,50,75$, and $95 \%$ of the peak intensity. The theoretical resolution element for each filter is shown in the lower-right corner of each panel. North is up and East is on the left. For a discussion on points A to C cf. Sec 7.1.
Fig 6). The angular size of the $\operatorname{disc}^{8}$ derived by the model (Eq. 1) is an upper limit to the real angular size.

The results of the fitting (Eq.1; Table 3.3) showed that the angular size (Gaussian FWHM) of II Lup's dusty circumstellar envelope increases at longer wavelengths: $\theta_{K s}=$ $34.4 \pm 2.2 \mathrm{mas}, \theta_{L^{\prime}}=69.2 \pm 1.5 \mathrm{mas}$, and $\theta_{M^{\prime}}=90.3 \pm 2.2$ mas. The observed visibilities suggest a flux contribution by an unresolved point source of approximately 35, 28 and $20 \%$ in $K s, L^{\prime}$ and $M^{\prime}$ respectively. However, this fitting procedure is not ideal when the visibilities vary with the azimuth, because essential information is averaged out with this procedure. In that sense, image reconstruction should reveal the true nature of the object.

\subsection{A comparison to Lopez et al.}

Lopez et al. (1993) observed II Lup in $1991(J D=2448434$, $\phi=0.17)$ using one-dimensional $\left(P A=292.5^{\circ}\right)$ speckleimaging on the ESO $3.6-\mathrm{m}$ telescope in $K(2.2 \mu \mathrm{m})$ and $L$ $(3.6 \mu \mathrm{m})$. Their data lacked azimuthal information, but was a first attempt at determining the angular size of II Lup. The authors modelled the data using a centro-symmetric circumstellar envelope, which provided an adequate fit to the spectral energy distribution of II Lup, but it could not produce a satisfactory fit for the visibilities (their Fig. 3). The stellar radius and the inner radius of the envelope at $2.2 \mu \mathrm{m}$ were 6 mas and 36 mas, respectively; or $3.5 \mathrm{AU}$ and $21 \mathrm{AU}$ at our adopted distance of $590 \mathrm{pc}$.

We have extracted the one-dimensional visibilities of Lopez et al. (1993) using the online tool Dexter ${ }^{9}$ and have overplotted them in Fig. 6 for reference (gray stars). The data of Lopez et al. agree in both bands with the SAM data within error bars up to a projected baseline of 2 metre. At $3.6 \mu \mathrm{m}$ the visibilities are lower than those of SAM by a decrement of about 0.15 for projected baselines ranging from 2 to 3 metres. This could be a result of the difference in de-projected baselines, as well as the different broadband filters used.

The angular size we derive for the $K s$ band is smaller than that of Lopez et al. (1993) $\left(\theta_{\mathrm{K}}\right.$, Lopez $\left.=72 \mathrm{mas}\right)$, and the estimated unresolved point-source flux contributions differ: $35 \%$ and $28 \%$ (this work), to less than $70 \%$ and $30 \%$ (Lopez et al.) in $K$ and $L$ respectively. This could be explained by (a) the longer baselines $\left(1.3 \leq B_{\mathrm{NACO}} \leq 6.9\right.$ to $0 \leq B_{\text {Lopez }} \leq 3$, in units of metres) and various azimuths offered by non-reduntant aperture masking, (b) the different phase at the time of observation ${ }^{10}$, or (c) a potential change in the morphology of the circumstellar envelope (e.g., IRC+10216, Stewart et al. 2016).

\section{IMAGE RECONSTRUCTION}

We present here our image reconstruction results using MACIM and MiRA. We have also tested a third method,

\footnotetext{
8 The results of the fitted uniform disc and Gaussian distributions were similar for the $K s$ band.

9 dc.zah.uni-heidelberg.de/sdexter

10 NACO observations were obtained at $J D=2455377.18$. If we adopt $t_{0}=2447174$ from Le Bertre (1992) and a period of 575 days, we find that the observations were carried at a phase $\phi=0.266$.
} 
BSMEM (Buscher 1994), which gave results similar to those of MACIM.

MACIM uses a point source, a uniform disc distribution or a binary as an initial model. We opted for an arbitrary uniform disc model that is unresolved by SAM/NACO with a size of 5 mas, that we assumed is the unresolved stellar diameter. We set the stellar flux contribution at 30-35\% and chose to multiply the visibility errors by a factor of 1.5 and the bispectrum errors by a factor of 2.0 to increase the range and to allow for any mis-calibration from the initial data reduction, and taking into account the correlations in the bispectrum data (M. Ireland, private communication). The $\chi^{2}$ of the reconstructed maps ranged from 0.83 to 1.0 , which suggests that we may have overcompensated for the errors.

The $K s$ map of II Lup is presented in the top panel of Figure 7. The majority of the emission $(\geq 60 \%$ of the peak intensity) originates from an area at the centre of the map. At lower emission levels, the shape of the circumstellar envelope is extended within the South-West quadrant and it clearly departs from spherical symmetry. A hooklike extension from the central source is evident. It spreads counter-clockwise from the South and extends West to approximately 100 mas from the origin. The southernmost extension is approximately 80 mas. Although this appears only at low levels of emission ( $0.5-1 \%$ of the peak), with the exception of three artefacts just south of II Lup, there is no other point of emission within the map above this level. For the adopted distance of $590 \mathrm{pc}$, the radius (16 mas) of the unresolved central source translates into 9.44 $\mathrm{AU}$ and the westernmost extension of the hook translates into 50.15 AU.

The morphology of the circumstellar envelope differs at longer wavelengths. The central source is once again unresolved in both maps (middle and bottom panels for the $L^{\prime}$ and $M^{\prime}$ maps, respectively, in Fig. 7). A small protrusion extends at a similar direction $\left(P . A . \simeq 235^{\circ}\right)$ in both maps. The overall morphology below $50 \%$ of the peak intensity is clearly non-spherically symmetric in both maps, and the shape of the circumstellar envelope is more extended directly North of the central source to approximately 120 and 150 milliarcseconds at the farthest for the $L^{\prime}$ and $M^{\prime}$ maps, respectively. In the $M^{\prime}$ map, the envelope appears to fan out at low peak-emission levels, however given the poor seeing of the $M^{\prime}$ observations (cf. Sec. 3), this reconstructed image is of lower fidelity.

The hook-like formation seen in the $K s$ map is absent at longer wavelengths. When the $K s$ map is convolved with a synthesized beam representative of the $L^{\prime}$ band observations ${ }^{11}$, the result indicates that the structure should have been partially resolved in $L^{\prime}$. Its absence can be explained by the lower resolution and the poorer $\mathrm{S} / \mathrm{N}$ of that specific night's observations.

We used MiRA by incorporating the tool SPARCO (Kluska et al. 2014) with the same initial parameters as in MACIM for consistency. The quadratic smoothness regularisation of MiRA with a weight $\mu=1 \mathrm{e}+9$ was used. Figure 8 shows the resulting image $\left(\chi^{2}=0.6\right)$. The overall shape of the structure agrees with the results of MACIM: the elongated envelope and the western clump are reproduced here.

11 That is, a Gaussian distribution with a FWHM of 60 mas.

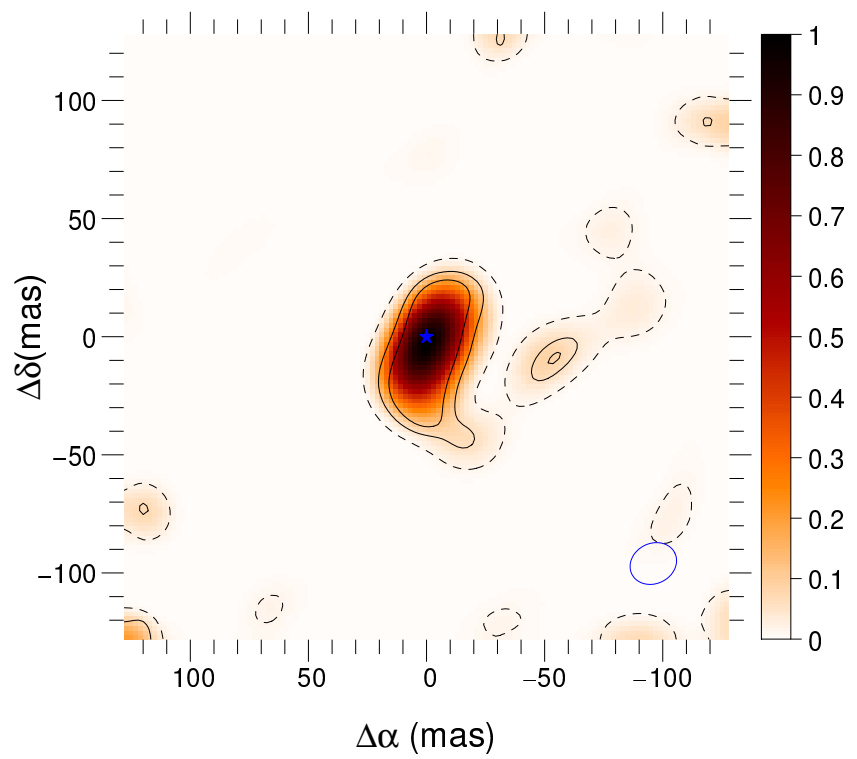

Figure 8. MiRA reconstruction for the $K s$ data. The position of the star is marked by the blue asterisk. The contours are 5, 3 (solid) and 1 sigma (dashed) significance contours. The blue ring indicates the resolution element.

At longer wavelengths, the resulting maps were almost identical to those produced by MACIM and therefore are not reproduced here.

\subsection{On the existence of a binary companion}

We now test the hypothesis of Feast et al. (2003) that II Lup may be a member of a binary system. SAM not only allows the recovery of diffraction-limited images, but the Fourier data can also be used to calculate the probability of locating a binary companion. In the past, SAM has enabled many direct detections of planetary companions in the $K s$ and $L^{\prime}$ bands (e.g., Lacour et al. 2011; Gauchet et al. 2016).

We have fitted a binary model to the SAM closure phases (two point sources). The modelled separation ranged between the diffraction limit and the field-of-view. SAM can achieve a contrast ratio up to 250, although the detection limit depends on the brightness of the primary (observed) $\operatorname{star}^{12}$. We chose to fit only the $K s$ dataset because (a) the best angular resolution is offered at that wavelength, and (b) the observations were obtained at better seeing conditions than those at the $L^{\prime}$ and $M^{\prime}$ that suffered from poor contrast.

The possible results of the simulations for the $K s$ dataset indicate the possible presence of a companion within the western tip of the hook-like formation seen in the $K s$ map (Fig. 7). The companion is placed at $48.48 \pm 5.87$ mas and $262^{\circ} \pm 3.6^{\circ}$ East-of-North from the central source, at a contrast of $29 \pm 4$, or a magnitude difference $\Delta K s=3.64$ $\left(\chi^{2}=127.5\right)$. At the adopted distance of $590 \mathrm{pc}$, the separation would be $28.6 \pm 3.46 \mathrm{AU}$. For this separation and assuming a circular orbit and two solar-mass stars, we derive an orbital period of approximately 108 years, which is 
significantly larger than the secondary periods derived here and by Feast et al.

This model is not fully convincing, because this companion would require a luminosity one thirtieth of that of the AGB star, or at about $100-200 L_{\odot}$ assuming a similar temperature. A luminous main sequence star can be excluded, as such a star would have evolved faster than II Lup. An evolved red giant is possible but unlikely, because such a companion would have an almost identical initial mass as II Lup.

If we assume that the 19.7 year secondary period is the orbital period, then the separation would be $9.19 \mathrm{AU}$, or else 15.6 mas at 590 pc. However, SAM/NACO would not be able to detect such a close companion to II Lup in $K s$ band. Such a detection might be possible with the best interferometric instrument, the VLTI, that can theoretically reach an angular resolution of 2 mas at its longest baseline with the auxiliary telescopes (e.g., GRAVITY/VLTI).

\section{DETERMINATION OF PHYSICAL PROPERTIES}

\subsection{Luminosity and effective temperature}

We now attempt to tentatively calculate the physical properties for II Lup using the primary period, $\log P=2.76$, the bolometric magnitudes found in the literature, and the modelled predictions for the stellar radius given in Table 2 .

Using the Period-Luminosity relation for carbon-rich Miras of Whitelock et al. (2006) $\left(M_{\mathrm{bol}}=-2.54 \log P+1.87\right)$, we derive an absolute bolometric magnitude, $M_{\text {bol }}=-5.141$ mag, which consequently provides a luminosity

$M_{\mathrm{bol}}-M_{\mathrm{bol}, \odot}=-2.5 \log \left(L_{*} / L_{\odot}\right)$

of approximately $9800 L_{\odot}$. From this derivation and the theoretical stellar radii, we calculate the effective temperatures, $T_{\text {eff }}$, given in Table 5 . Although our derived luminosity differs from the models found in the literature, the range of the derived effective temperatures is congruent with the range of the modelled effective temperatures (22002800 K; Danilovich et al. 2015; Ramstedt \& Olofsson 2014; De Beck et al. 2010; Dehaes et al. 2007; Schöier et al. 2007; Ryde et al. 1999; Groenewegen et al. 1998). The only exception is the effective temperature $(2082 \mathrm{~K})$ derived for the stellar radius ${ }^{13}$ of Lopez et al. (1993); the temperature adopted by Lopez et al. was $2200 \mathrm{~K}$.

\subsection{Dust temperature}

As a comparison, we estimate the temperature of the circumstellar envelope in the $K s$ band using the angular size derived from our observations, $\theta_{K s}=34.4 \pm 2.2$ mas, the apparent bolometric magnitude $m_{\mathrm{bol}}=3.71$, and the relation of Bedding et al. (1997),

$\log T=4.22-0.1 m_{\text {bol }}-0.5 \log \theta_{K s}$

Although the relation above should provide the effective temperature of a star, the angular size derived from the $\mathrm{SAM} / \mathrm{NACO}$ observations does not correspond to the stellar

13 The angular radius of 6 mas projected at a distance of $590 \mathrm{pc}$.

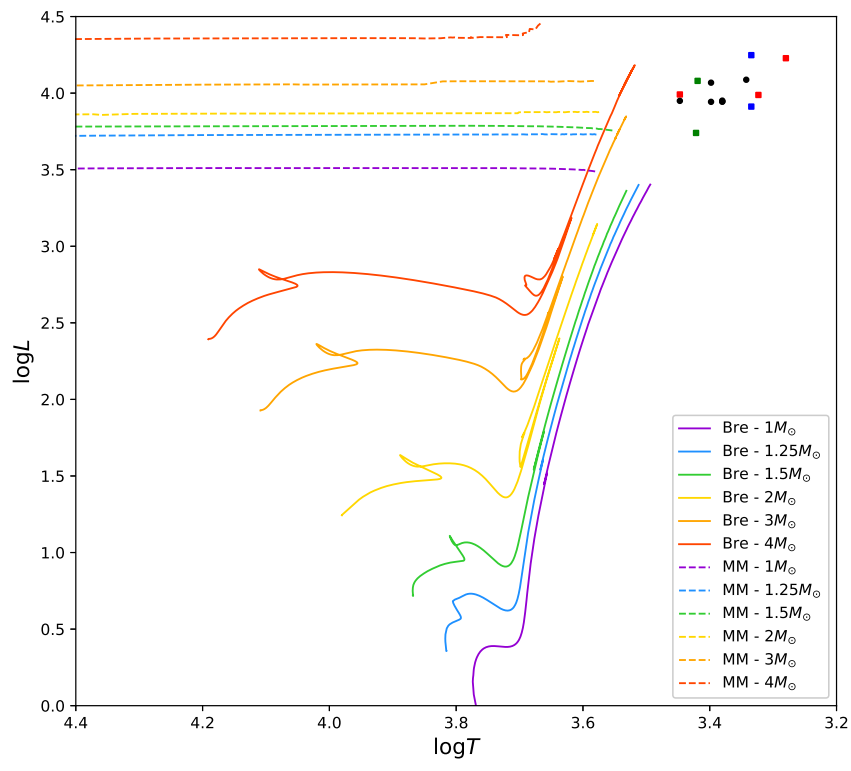

Figure 9. Evolutionary tracks of intermediate-mass stars $\left(1-4 \mathrm{M}_{\odot}\right)$ from Bressan et al. (2012) (bold lines) and from Miller Bertolami (2016) (dashed lines). The physical parameters of II Lup $(\log T, \log L$; black circles) were extracted from the theoretical models found in the literature (Danilovich et al. 2015; Ramstedt \& Olofsson 2014; Schöier et al. 2013; De Beck et al. 2010; Dehaes et al. 2007; Groenewegen et al. 1998; Lopez et al. 1993). Three additional carbon-rich AGB stars are overplotted on the H-R diagrams for comparison: R Scl (green squares, Wittkowski et al. 2017; Bergeat et al. 2002), V Hya (blue squares, Groenewegen et al. 2002b; Bergeat et al. 2002) and CW Leo (red squares, Ramstedt \& Olofsson 2014; Groenewegen et al. 2002b; Bergeat et al. 2002). For a further description, see main text (cf. Sec. 6.3).

diameter. The derived temperature, $T \sim 1200 \mathrm{~K}$, is that of the dust enclosed within said angular size.

\subsection{Mass}

Ryde et al. (1999) have characterized II Lup as a "highly evolved carbon star" with a significantly lower carbon isotopic ratio $\left(4 \leq{ }^{12} \mathrm{C} /{ }^{13} \mathrm{C} \leq 10\right.$, see also Woods et al. 2003; De Beck et al. 2010; Ramstedt \& Olofsson 2014) as opposed to typical carbon stars ( 30; Nyman et al. 1993; Greaves \& Holland 1997). According to the authors, the lower isotopic ratio could suggest that II Lup may have been a $5-8 \mathrm{M}_{\odot}$ star, where hot-bottom burning has been suppressed therefore preventing the star from becoming oxygenrich. Here, we use three different methods to estimate the mass of II Lup based on the theoretical stellar radii in Table 2 . These methods are described below.

\subsubsection{Evolutionary tracks}

We attempt to place II Lup on a Hertzsprung-Russell diagram. We selected evolutionary tracks for stars of solar metallicity and with initial masses between $1-4 \mathrm{M}_{\odot}$ from Bressan et al. (2012) and from Miller Bertolami (2016) to cover the evolutionary sequences until and beyond the AGB phase, respectively. These evolutionary tracks are shown in 
Table 5. Physical properties of II Lup

\begin{tabular}{cccc}
\hline$R_{*}[\mathrm{AU}]$ & $T_{\text {eff }}[\mathrm{K}]$ & \multicolumn{2}{c}{$M\left[M_{\odot}\right]$} \\
& & $(1)$ & $(2)$ \\
\hline 1.86 & 2869 & 1.95 & 1.87 \\
2.32 & 2571 & 3.76 & 2.99 \\
2.52 & 2466 & 4.83 & 3.59 \\
2.54 & 2456 & 4.95 & 3.66 \\
2.66 & 2399 & 5.71 & 4.05 \\
3.00 & 2246 & 8.46 & 5.38 \\
3.54 & 2082 & 13.34 & 7.48 \\
\hline
\end{tabular}

Methods: (1) Fox \& Wood (1982);

(2) van Leeuwen et al. (1997)

Figure 9. The physical parameters of II Lup $(\log T, \log L$; black circles) were extracted from the models found in the literature (Danilovich et al. 2015; Ramstedt \& Olofsson 2014; Schöier et al. 2013; De Beck et al. 2010; Dehaes et al. 2007; Groenewegen et al. 1998; Lopez et al. 1993). As a comparison, we show the locations of three other carbonrich AGB stars on these $\mathrm{H}-\mathrm{R}$ diagram: $\mathrm{R}$ Scl (green squares, Wittkowski et al. 2017; Bergeat et al. 2002), V Hya (blue squares, Groenewegen et al. 2002b; Bergeat et al. 2002) and CW Leo (red squares, Ramstedt \& Olofsson 2014; Groenewegen et al. 2002b; Bergeat et al. 2002). Despite the uncertainties in the physical properties of the theoretical models, the $\mathrm{H}-\mathrm{R}$ diagram indicates that II Lup is placed near the tip of the AGB. We could therefore deduce that the initial stellar mass is $1.5 \pm 0.5 \mathrm{M} \odot$, however we must point out that the Bressan et al. (2012) evolutionary tracks terminate at the tip of the RGB for stars below $2 \mathrm{M}_{\odot}$ and at the beginning of the TP-AGB phase at higher masses.

\subsubsection{The method of Fox \& Wood (1982)}

Following the method described in Soszyński \& Wood (2013), we calculated the extinction-corrected Wesenheit index in the near-infrared, $W_{\mathrm{JK}}$, using the the 2MASS photometry and the distance of Pietrzyński et al. (2013) for the Large Magellanic Cloud. The resulting value $\left(W_{\mathrm{JK}}=\right.$ $8.53 \pm 0.05)$ places II Lup near the tip of the fundamental mode of carbon-rich stars in Figure 1 of Soszyński \& Wood (2013).

Therefore, if we assume that the primary period of II Lup is on the fundamental mode, and following the pulsation equation of Fox \& Wood (1982), the stellar mass is given by

$M=\left(\frac{Q}{P}\right)^{2} R^{3}$

where $M$ and $R$ are given in solar units, and $Q \sim 0.1$ for the fundamental mode (based on the predictions for $0.8 \lesssim M \lesssim 3.0 \mathrm{M}_{\odot}$ of Fox \& Wood 1982). Using this formula we can tentatively estimate the mass of II Lup for the given stellar radii (Table 5). The range of the derived values is quite large, however the calculations were based on parameters with large uncertainties, such as the theoretical stellar radii and the distance.

\subsubsection{The method of van Leeuwen et al. (1997)}

We now attempt to estimate the stellar mass using the relation for the fundamental mode of van Leeuwen et al. (1997):

$\log M=(1.949 \log R-2.07-\log P) / 0.9$

and the resulting values are tabulated in Table 5 .

The initial mass of a carbon-rich AGB star can be within the range of $1-4 \mathrm{M}_{\odot}$, but this is highly dependent on the metallicity (Karakas \& Lugaro 2016; Miller Bertolami 2016). However, the stellar masses derived by the method of Fox \& Wood (1982) for the given stellar radii vary by a large factor. The last two values are much higher than the mass limit for intermediate-mass stars. Therefore, only theoretical radii below 2.5 AU would provide realistic stellar masses for II Lup based on the methods of Fox \& Wood (1982) and van Leeuwen et al. (1997), i.e. $\underset{\sim}{<} 3.6 \mathrm{M}_{\odot}$.

\section{THE CIRCUMSTELLAR ENVIRONMENT OF II LUP}

\subsection{On a putative spiral formation detected by NACO}

Let us now consider the timescale of the expansion of the structure in the $K s$ map (Fig. 7). We adopt the gas expansion velocity $v_{\exp }=22.2 \mathrm{~km} \mathrm{~s}^{-1}$ (Nyman et al. 1993), and assume that the dust velocity is similar and that the expansion is linear. We select three locations at the $0.5 \%$ peak-emission level on the $K s$ map (Fig. 7): one directly East of the central source (hereby point A), one directly South (point B), and one directly West (point C). Following our previous assumptions and again adopting a distance of $590 \mathrm{pc}$, the timescales for the expansion of the dust up to points A, B and C are 4, 8.9 and 11.7 years respectively. However, if the ejecta were expanding radially and the expansion was spherically symmetric, the ejecta would not have been located only in the lower-right quadrant of the map.

We now assume that the counter-clockwise hook-like extension in the $K s$ map of II Lup (Fig. 7) is the inner arm of a spiral. Spiral formations are not uncommon, however only a handful of spirals have been detected around AGB stars in the last decade. These characteristic examples are: AFGL 3068 (Kim et al. 2017; Mauron \& Huggins 2006), R Scl (Maercker et al. 2012), RW LMi (Monnier et al. 2000; Kim et al. 2013), and CW Leo (Decin et al. 2015). All four stars are carbon-rich like II Lup. Observations suggest that two oxygen-rich stars, Mira (Ramstedt et al. 2014) and W Aql (Mayer et al. 2013), and one S-type star (the possibly triple system of $\pi^{1}$ Gru, Mayer et al. 2014), also indicate the presence of a spiral. All of the examples mentioned above are known binaries or strongly expected to belong in a binary system.

Following these assumptions, if we consider that the coil of the putative spiral reached the western tip of the hook (point C) within 12 years, it could complete a counterclockwise rotation over four quadrants within 40 years. However, the full extent of the influence of binarity in such spirals is seen at larger spatial scales $\left(\underset{\sim}{>10^{\prime \prime}}\right)$ compared to the field-of-view of SAM/NACO. We cannot exclude a longer period considering many AGB stars with spirals have longer 


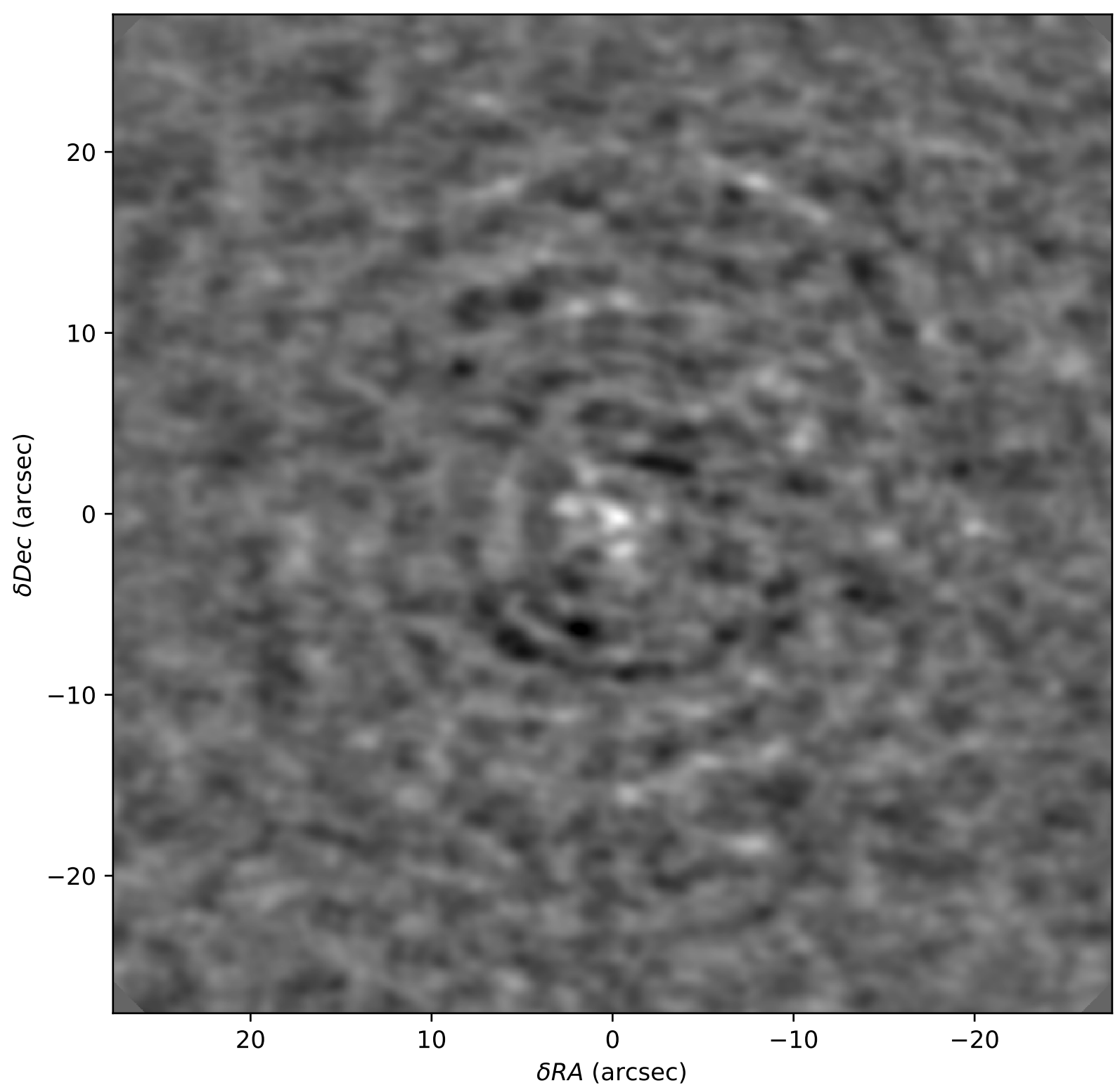

Figure 10. ALMA CO $(J=1-0)$ channel map. The large scale structure extends approximately $23^{\prime \prime}$ from the central star.

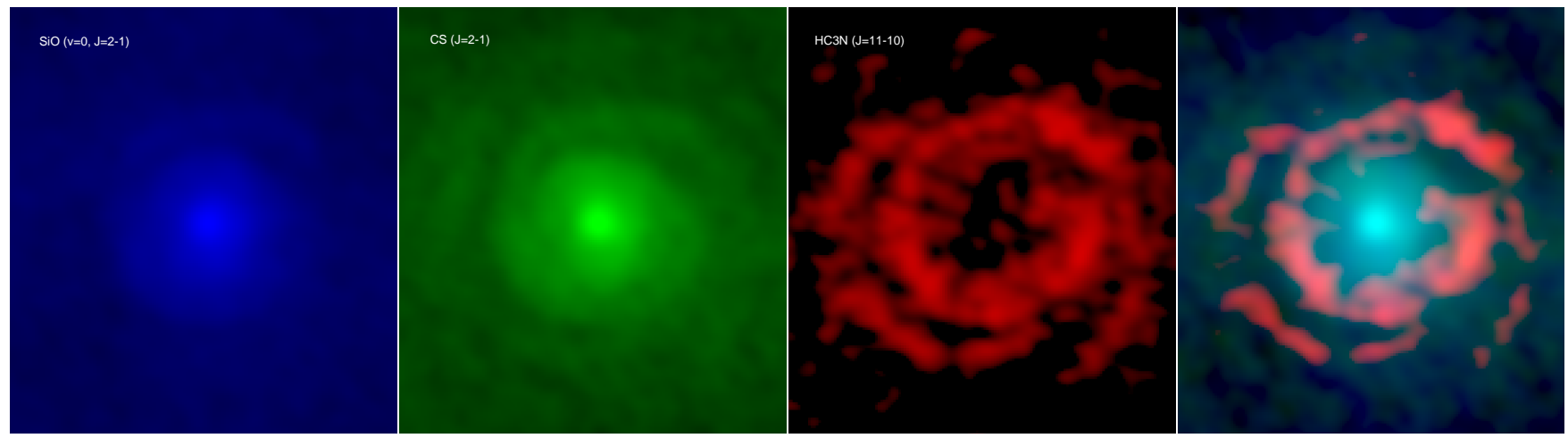

Figure 11. The inner coils of II Lup's spiral shown in three different molecules, namely $\mathrm{SiO}, \mathrm{CS}$ and $\mathrm{HC}_{3} \mathrm{~N}$ from left to right. The last panel shows a false-colour image of the spiral based on these three molecules. North is up and east is on the left. 
Table 6. ALMA results

\begin{tabular}{llcc}
\hline Molecule & Transition & $\begin{array}{c}\text { Frequency } \\
{[\mathrm{GHz}]}\end{array}$ & $\begin{array}{c}D_{\text {area }}(*) \\
\text { [arcsec] }\end{array}$ \\
\hline${ }^{12} \mathrm{CO}$ & $J=1-0$ & 115.276 & $<56$ \\
$\mathrm{HC}_{3} \mathrm{~N}$ & $J=11-10$ & 100.083 & $\sim 14.2$ \\
$\mathrm{CS}$ & $J=2-1$ & 97.9873 & $\sim 10.4$ \\
$\mathrm{SiO}$ & $v=0, J=2-1$ & 86.8526 & $\sim 10$ \\
\hline
\end{tabular}

$(*)$ : diameter of the circumstellar envelope.

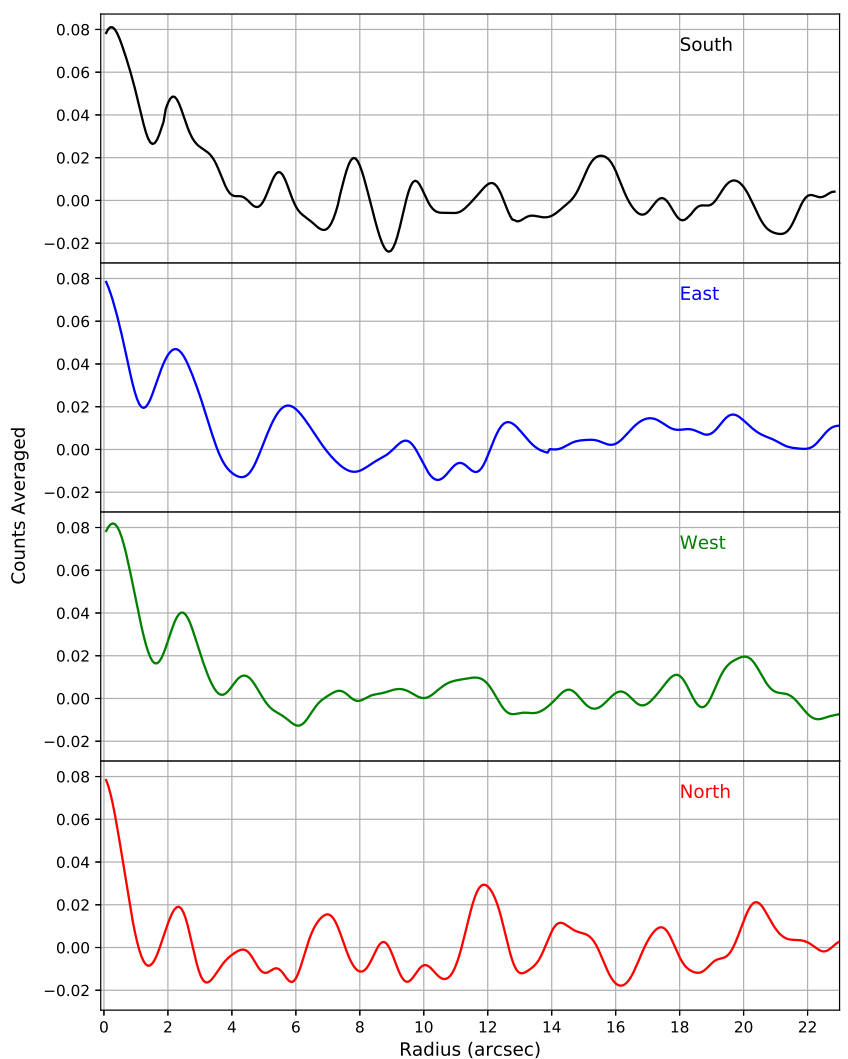

Figure 12. Radial profile projection from the position of II Lup (2MASS coordinates) and extending up to $23^{\prime \prime}$ in all cardinal points to illustrate the location of the spiral arms in each direction.

orbital periods (e.g., R Scl: 350 years (Maercker et al. 2012), AFGL 3068: 830 years (Mauron \& Huggins 2006)), and we demonstrate this argument in the following sections.

\subsection{Tracing the circumstellar envelope with ALMA}

To test the validity of the asymmetries detected with aperture masking, we retrieved an ALMA data set from the archive (cf. Sec. 3.3). The observations covered a number of spectral lines, at sub-arcsecond resolution. Images of four detected emission lines are shown in Figures 10 and 11. The lines are from the molecules $\mathrm{CO}, \mathrm{SiO}, \mathrm{CS}$, and $\mathrm{HC}_{3} \mathrm{~N}$ (Table 6$)$. $\mathrm{SiO}$ and $\mathrm{CS}$ are detected from the innermost region: these are molecules that exist in the inner wind. $\mathrm{HC}_{3} \mathrm{~N}$ forms a little further out and traces the inner envelope but not the near-stellar region. CO is detected throughout the envelope.

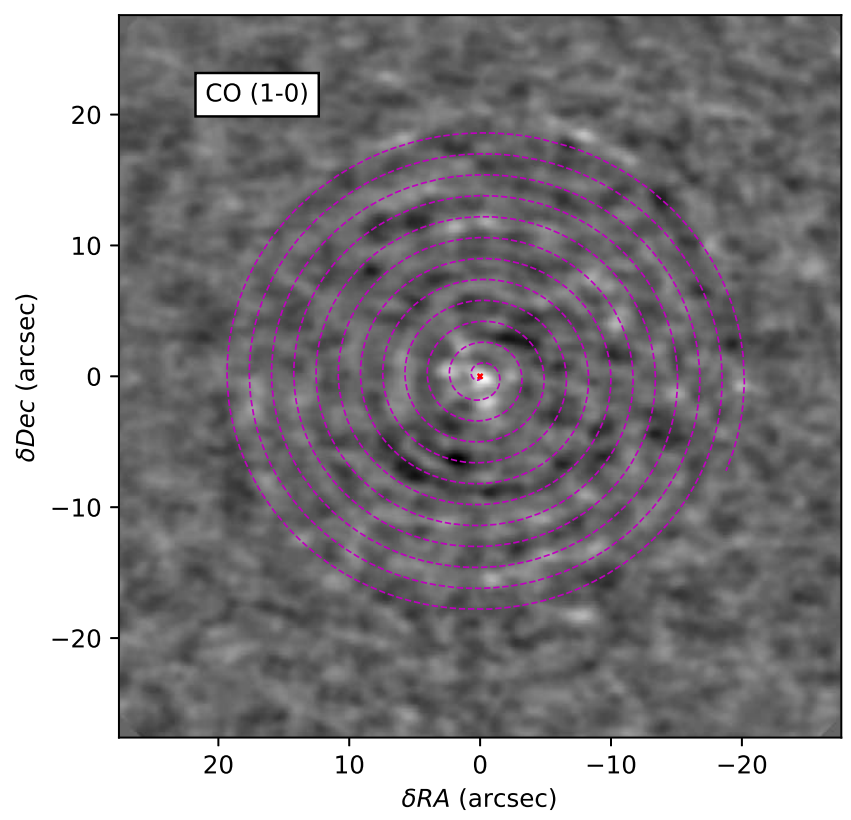

Figure 13. A counter-clockwise spiral with a 1.7" arm separation overplotted on the CO channel map of Fig.10. The red star marks the position of II Lup in the Gaia system.

The images show the spectral channel corresponding to the central velocity.

We find that the circumstellar, molecular envelope of II Lup extends as far out as $23^{\prime \prime}$ from the central source in the CO (1-0) transition. The most striking discovery from this dataset is a spiral traced by the carbon monoxide gas (Fig. 10). The spiral arms appear patchy and faint at places, but the overall structure is evident in the CO map. The ALMA CO image is significantly affected by missing short spacings. It is therefore sensitive to small-scale structure which is dominated by the spiral pattern, but the large structure is resolved out.

The last panel of Fig.11 shows a false-colour image composed of three molecules ( $\mathrm{SiO}, \mathrm{CS}$, and $\mathrm{HC}_{3} \mathrm{~N}$ ). Only one northern coil extending clock-wise can be seen in the $\mathrm{SiO}$ and CS channel maps, while the overall emission is dominated by the central core. The coil is an exact match in both molecules. On the other hand, the $\mathrm{HC}_{3} \mathrm{~N}$ channel map shows the absence of a central source, thus indicating that the molecular emission arises only from the circumstellar envelope. The most striking feature in the $\mathrm{HC}_{3} \mathrm{~N}$ map is the distinct separation of the structure in two different arms.

All four images show a clear spiral signature. The location of the windings depends on the velocity: the selected channels are those where the windings are located furthest out, as expected at the systemic velocity for an expanding envelope. Because the observed location changes with the selected velocity, averaging of spectral channel reduces the contrast of the spiral.

From the position of II Lup (based on the 2MASS coordinates), we obtain radial cuts in the CO image in four directions: south, north, east and west. The profiles are shown in Fig. 12. The profiles show clear peaks where the cuts crosses a spiral winding: a total of 12 windings were detected, out to the largest distance of $23^{\prime \prime}$. The peaks were measured to obtain their distance from the star. We measured the sepa- 

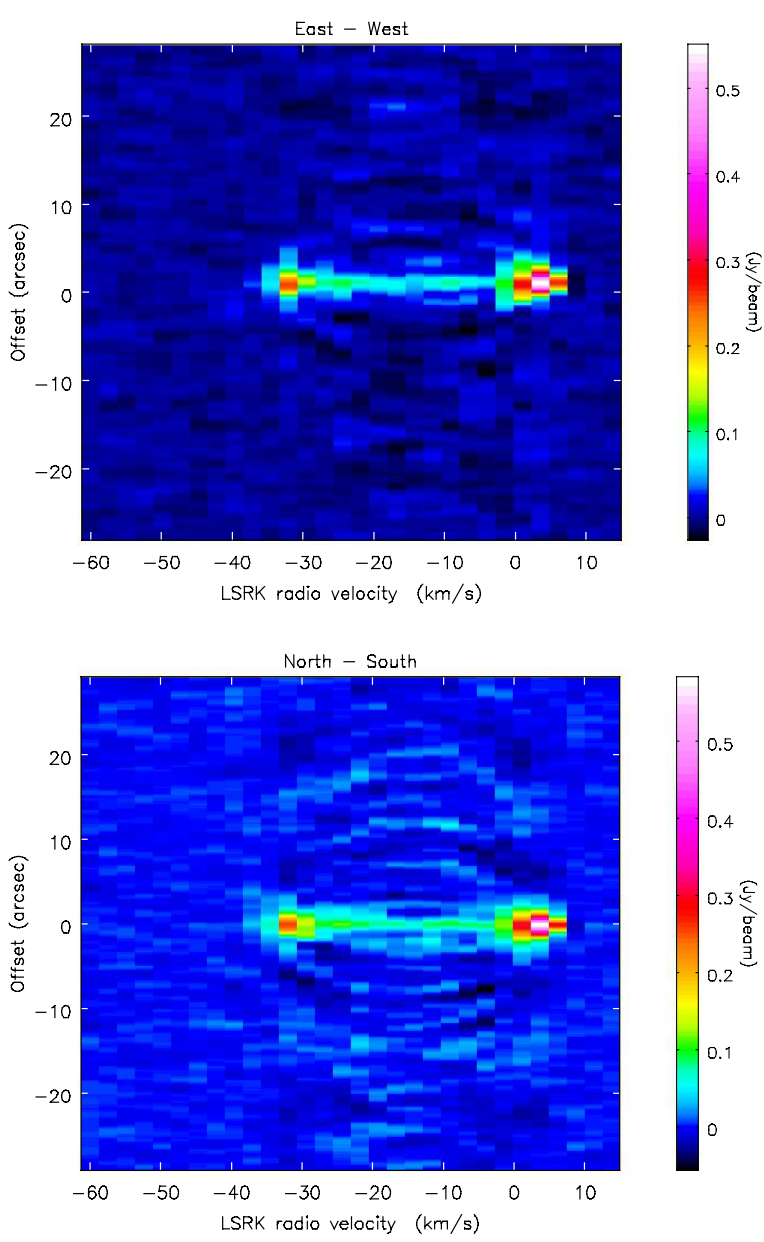

Figure 14. Position-velocity diagrams along the east-west (top) and north-south axis (bottom) through the centre of the CO map.

rations for each of these peaks. For each cut, we calculated the median separation: $1.565^{\prime \prime}, 1.595^{\prime \prime}, 1.69^{\prime \prime}$ and $1.75^{\prime \prime}$, for south, north, east and west, respectively. The separation is slightly larger in the east-west direction than in the northsouth, suggesting there may be some flattening of the spiral. However this difference is marginal. We find a median separation of 1.7 arcsec.

Figure 14 presents the position-velocity (P-V) diagrams along the north-south and east-west axis of the CO (1-0) map (Fig. 10). At a first inspection, the P-V diagrams show a narrow bar of emission at the centre with enhanced features at $+5 \mathrm{~km} \mathrm{~s}^{-1}$ and $-35 \mathrm{~km} \mathrm{~s}^{-1}$ corresponding to the core of Fig. 10. At lower intensity levels (below $0.1 \mathrm{Jy} / \mathrm{beam}$ ) arc features are visible ${ }^{14}$ extending up to about $\pm 20^{\prime \prime}$ along the north-south axis (bottom panel, Fig. 14). These features are less prominent in the east-west P-V diagram (top panel, Fig. 14), although two arcs are visible within $\pm 10^{\prime \prime}$ from the central bar.

We compare these diagrams to the work of Homan et al. (2015), who simulated CO emission from the circumstellar envelopes of AGB stars, and in particular to their modelled

14 For a better view we refer to the electronic version of this figure.
$\mathrm{P}-\mathrm{V}$ diagrams. We find that the $\mathrm{P}-\mathrm{V}$ diagrams of Figure 14 (this work) are somewhat similar to both those in Fig. 9 of Homan et al. (2015) for their model of an edge-on, narrow spiral with a low mass-loss wind and a more massive homogeneous-to-spiral wind outflow, as well as those in their Fig. 11 for a "shell spiral" with a low mass-loss wind and an equal homogeneous-to-spiral wind outflow ${ }^{15}$. As shown here in Fig. 10, the structure does not appear to be edge-on and the east-west P-V diagram in Fig.14 is not "S-shaped", therefore the first case of Homan et al. (2015) for the narrow spiral might not apply here. The models of Homan et al. (2015) for their "shell spiral" indicate slightly similar P-V diagrams with a bright, narrow strip at the centre of the $\mathrm{P}-\mathrm{V}$ diagram (their Fig.11) that is not seen here (this work, Fig. 14).

This is a tentative comparison since in the work of Homan et al. (2015) the P-V diagrams were modelled for a star similar to CW Leo and for the CO $J=3-2$ transition which traces the inner wind unlike the CO $J=1-0$ transition. Moreover, the mass loss of II Lup is significantly higher than the one in the low mass-loss wind model of Homan et al. (2015). As a last point, the simulated observations of Homan et al. (2015) for the ALMA C34-7 configuration (their Fig.19, panel D) does not match the observations presented here that were obtained with a similar configuration in 2015. Further observations will be required to re-visit the complexity of the circumstellar envelope of II Lup as seen by ALMA.

As a last step, we follow the analysis of Mayer et al. (2013) to determine initial parameters for the putative spiral around II Lup, and use their equations for the arm separation,

$\rho=v_{\exp } \cdot P \cdot \pi$

where here $\rho$ is the arm separation given above, $P$ is the orbital period and $\pi$ is the parallax (for the adopted distance of $590 \mathrm{pc}$ ). We derive an orbital period of about 128 years.

\subsection{Comparing the results of SAM/NACO and ALMA.}

Figure 13 shows the spiral with the $1.7^{\prime \prime}$ arm separation superposed on the CO image. The starting angle of the spiral was used as a free parameter. The spiral was given the slight flattening indicated by the $\mathrm{CO}$ data. It provides an acceptable fit. There is also some complexity which goes beyond a simple spiral structure. This is expected from models (Mohamed \& Podsiadlowski 2012) which show that in binary motion, there is a main spiral caused by the movement of the centre of mass loss, but there is a second, counter spiral caused by interaction between the wind and the companion.

It is possible that the secondary arm, or a portion of it near the mass-losing star, was detected by SAM/NACO observations. In Figures 7 and 8, the envelope is shown to

15 Homan et al. (2015) distinguish a "narrow" from a "shell spiral" depending on their definition of the spiral's opening angle, that is how their model spirals open with the respect to the orbital plane. A small opening angle results in a "narrow" spiral, while an opening angle of $180^{\circ}$ gives a "shell spiral". For a more in-depth description, we refer to Homan et al. (2015) and their Sect.2.1.2. 
extend south-west within $190^{\circ}<$ P.A. $<235^{\circ}$, which is not clearly aligned with the spiral traced by the molecular gas. The models of Mohamed \& Podsiadlowski (2012) suggest no structure inside the stellar orbit, which would imply that the binary companion should not be further out than component 'C' (Ks map of Fig. 7). It is of course still possible that component ' $\mathrm{C}$ ' is the binary, or at least emission associated with it. In that case, the luminosity of the companion ought to be less than $200 \mathrm{~L}_{\odot}$ or else it would have been clearly detected by SAM/NACO. If we assume that the initial mass of II Lup was within $2.5 \pm 1 \mathrm{M}_{\odot}$, as calculated in Sec.6.3, then a 128 -year period would imply $m_{2} \lesssim 1 \mathrm{M}_{\odot}$. Since the detected structure appears to be seen nearly pole-on, this hypothesis can be tested by further observations in the near infrared.

The indication of a larger separation would be consistent with a reduction in the mass of the primary star or an increase in the wind speed. However, the indication is only tentative. We find that the ALMA data provides strong support for the existence of a spiral structure, which implies that binarity is the main shaping mechanism of the mass loss, therefore the effects of a companion's orbital motion is the most likely explanation of the asymmetries seen in the aperture masking data.

\section{CONCLUSIONS}

The carbon star II Lup is known to exhibit an obscuration event in addition to a primary period of 575 days. According to Feast et al. (2003) this can be explained either by the ejection of dust clumps or by the presence of a companion. After re-analysing the infrared lightcurve of II Lup, we confirm a secondary period of approximately 19 years. However, due to the incompleteness of the sample, the true secondary period may be significantly longer. We attempted to estimate the physical parameters of II Lup (luminosity, effective temperature and mass), however we find that the results are inconclusive due to the large uncertainties of the measurements used. Nevertheless, we obtain a dust temperature of $\sim 1200 \mathrm{~K}$ from the $K s$ angular size of II Lup.

This work presents the first-ever images of the dusty envelope of II Lup in near-infrared and sub-mm wavelengths. We have used an aperture masking technique to obtain diffraction limited images from a single-dish telescope in $K s$, $L^{\prime}$ and $M^{\prime}$. The interferometric data revealed that:

(i) the angular size of the source, $\theta_{K s}$, is less than 35 mas, and therefore the stellar radius is much smaller than $20 \mathrm{AU}$ (assuming a distance of $590 \mathrm{pc}$ ),

(ii) the morphology of the circumstellar envelope is nonspherical and that its shape is hook-like at low emission levels (Ks map, Fig. 7), possibly resembling the inner coil of a spiral, or associated with a counter-spiral shaped by orbital motion (e.g., Mohamed \& Podsiadlowski 2012),

(iii) the morphology of the envelope is different at longer wavelengths ( $L^{\prime}$ and $M^{\prime}$ ), where the envelope expands directly north from the central source. However, due to a lower resolution and signal-to-noise at those wavelengths, the fidelity of those images is lower and we can conclude that the envelope is smaller than 200 mas in these bands.

Our analysis of the archived ALMA channel maps from
${ }^{12} \mathrm{CO}(J=1-0), \mathrm{SiO}(v=0, J=1-0), \mathrm{CS}(J=2-$ 1) and $\mathrm{HC}_{3} \mathrm{~N}(J=11-10)$, reveals the true extent of the non-spherical circumstellar envelope and most importantly hints at a large-scale spiral structure in all maps. From the spacing of the spiral arms in the CO map $\left(\sim 1.7^{\prime \prime}\right)$, we derive an approximate orbital period of 128 years. A study of the higher CO transitions tracing the inner wind of II Lup would be beneficial in determining the history of the mass loss process.

Given the complexity of this object we suggest new observations of the circumstellar environment of II Lup in order to compare the morphology at different epochs. We strongly recommend (a) further monitoring of its variability in the visual and near-infrared, and (b) a polarimetric and spatio-kinematic study of its non-spherical dusty environment with large-aperture telescopes (e.g., GRAVITY/VLTI and SPHERE/VLT).

\section{ACKNOWLEDGEMENTS}

We acknowledge with thanks the assistance of the NACO team during the challenging observations. We thank Patrick Lenz for his assistance with PERIOD04. FL acknowledges support from the Hong Kong University Postdoctoral Fellowships scheme and the FWF AP23006 project (PI: Josef Hron). JK acknowledges support from the Philip Leverhulme Prize (PLP-2013-110, PI: Stefan Kraus), and from the research council of the KU Leuven under grant number $C 14 / 17 / 082$. This research has made use of Astropy ${ }^{16}$, a community-developed core Python package for Astronomy (Astropy Collaboration et al. 2013), and "Aladin sky atlas" developed at CDS, Strasbourg Observatory, France. We have also used the SIMBAD database and the VizieR catalogue access tool operated at CDS, Strasbourg (France), as well as NASA's Astrophysics Data System Bibliographic Services. We thank the European Southern Observatory for allocating time on NACO and VISIR for this project. This paper makes use of the following ALMA data: ADS/JAO.ALMA\#2013.1.00070.S. ALMA is a partnership of ESO (representing its member states), NSF (USA) and NINS (Japan), together with NRC (Canada), MOST and ASIAA (Taiwan), and KASI (Republic of Korea), in cooperation with the Republic of Chile. The Joint ALMA Observatory is operated by ESO, AUI/NRAO and NAOJ. This research used the facilities of the Canadian Astronomy Data Centre operated by the National Research Council of Canada with the support of the Canadian Space Agency. This research was made possible through the use of the AAVSO Photometric All-Sky Survey (APASS), funded by the Robert Martin Ayers Sciences Fund. Based on observations made with the NASA/ESA Hubble Space Telescope, and obtained from the Hubble Legacy Archive, which is a collaboration between the Space Telescope Science Institute (STScI/NASA), the Space Telescope European Coordinating Facility (ST-ECF/ESA) and the Canadian Astronomy Data Centre (CADC/NRC/CSA).

16 www.astropy.org 


\section{REFERENCES}

Alfonso-Garzón J., Domingo A., Mas-Hesse J. M., Giménez A., 2012, A\&A, 548, A79

Astropy Collaboration et al., 2013, A\&A, 558, A33

Baron F., et al., 2012, in Optical and Infrared Interferometry III. p. $84451 \mathrm{E}$ (arXiv:1207.7141), doi:10.1117/12.924907

Bedding T. R., Zijlstra A. A., von der Luhe O., Robertson J. G., Marson R. G., Barton J. R., Carter B. S., 1997, MNRAS, 286, 957

Bedding T. R., Zijlstra A. A., Jones A., Marang F., Matsuura M., Retter A., Whitelock P. A., Yamamura I., 2002, MNRAS, 337,79

Bergeat J., Knapik A., Rutily B., 2002, A\&A, 390, 967

Bladh S., Höfner S., Aringer B., Eriksson K., 2015, A\&A, 575, A105

Bonnarel F., et al., 2000, A\&AS, 143, 33

Bressan A., Marigo P., Girardi L., Salasnich B., Dal Cero C., Rubele S., Nanni A., 2012, MNRAS, 427, 127

Buscher D. F., 1994, in Robertson J. G., Tango W. J., eds, IAU Symposium Vol. 158, Very High Angular Resolution Imaging. p. 91

Castro-Carrizo A., et al., 2010, A\&A, 523, A59

Clayton G. C., et al., 2011, ApJ, 743, 44

Cox N. L. J., et al., 2012, A\&A, 537, A35

Crause L. A., Lawson W. A., Henden A. A., 2007, MNRAS, 375,301

Cutri R. M., et al., 2003, VizieR Online Data Catalog, 2246

Danilovich T., et al., 2015, A\&A, 581, A60

De Beck E., Decin L., de Koter A., Justtanont K., Verhoelst T., Kemper F., Menten K. M., 2010, A\&A, 523, A18

Decin L., Richards A. M. S., Neufeld D., Steffen W., Melnick G., Lombaert R., 2015, A\&A, 574, A5

Dehaes S., Groenewegen M. A. T., Decin L., Hony S., Raskin G., Blommaert J. A. D. L., 2007, MNRAS, 377, 931

ESA ed. 1997, The HIPPARCOS and TYCHO catalogues. Astrometric and photometric star catalogues derived from the ESA HIPPARCOS Space Astrometry Mission ESA Special Publication Vol. 1200

Epchtein N., Le Bertre T., Lepine J. R. D., Marques Dos Santos P., Matsuura O. T., Picazzio E., 1987, A\&AS, 71, 39

Feast M. W., Whitelock P. A., Marang F., 2003, MNRAS, 346,878

Fox M. W., Wood P. R., 1982, ApJ, 259, 198

Gaia Collaboration et al., 2016, A\&A, 595, A2

Gaia Collaboration Brown A. G. A., Vallenari A., Prusti T., de Bruijne J. H. J., Babusiaux C., Bailer-Jones C. A. L., 2018, preprint, p. arXiv:1804.09365 (arXiv:1804.09365)

Gauchet L., Lacour S., Lagrange A.-M., Ehrenreich D., Bonnefoy M., Girard J. H., Boccaletti A., 2016, A\&A, 595, A31

Greaves J. S., Holland W. S., 1997, A\&A, 327, 342

Groenewegen M. A. T., Whitelock P. A., Smith C. H., Kerschbaum F., 1998, MNRAS, 293, 18

Groenewegen M. A. T., Baas F., Blommaert J. A. D. L., Stehle R., Josselin E., Tilanus R. P. J., 1999, A\&AS, 140, 197

Groenewegen M. A. T., Sevenster M., Spoon H. W. W., Pérez I., 2002a, A\&A, 390, 501

Groenewegen M. A. T., Sevenster M., Spoon H. W. W., Pérez I., 2002b, A\&A, 390, 511

Groenewegen M. A. T., et al., 2011, A\&A, 526, A162

Guandalini R., Busso M., Ciprini S., Silvestro G., Persi P., 2006, A\&A, 445, 1069

Hajduk M., Zijlstra A. A., Gesicki K., 2008, A\&A, 490, L7

Höfner S., Gautschy-Loidl R., Aringer B., Jørgensen U. G., 2003, A\&A, 399, 589

Homan W., Decin L., de Koter A., van Marle A. J., Lombaert R., Vlemmings W., 2015, A\&A, 579, A118

Ireland M. J., Monnier J. D., Thureau N., 2006, in Society of
Photo-Optical Instrumentation Engineers (SPIE) Conference Series. p. 62681T, doi:10.1117/12.670940

Jeffers S. V., Min M., Waters L. B. F. M., Canovas H., Rodenhuis M., de Juan Ovelar M., Chies-Santos A. L., Keller C. U., 2012, A\&A, 539, A56

Karakas A. I., Lugaro M., 2016, ApJ, 825, 26

Karovicova I., Wittkowski M., Ohnaka K., Boboltz D. A., Fossat E., Scholz M., 2013, A\&A, 560, A75

Kervella P., Homan W., Richards A. M. S., Decin L., McDonald I., Montargès M., Ohnaka K., 2016, A\&A, 596, A92

Kim H., Hsieh I.-T., Liu S.-Y., Taam R. E., 2013, ApJ, 776, 86

Kim H., Trejo A., Liu S.-Y., Sahai R., Taam R. E., Morris M. R., Hirano N., Hsieh I.-T., 2017, Nature Astronomy, 1, 0060 EP

Kluska J., et al., 2014, A\&A, 564, A80

Knapp G. R., Dobrovolsky S. I., Ivezić Z., Young K., Crosas M., Mattei J. A., Rupen M. P., 1999, A\&A, 351, 97

Lacour S., Tuthill P., Amico P., Ireland M., Ehrenreich D., Huelamo N., Lagrange A.-M., 2011, A\&A, 532, A72

Lagadec E., et al., 2011, MNRAS, 417, 32

Landsman W. B., 1993, in Hanisch R. J., Brissenden R. J. V., Barnes J., eds, Astronomical Society of the Pacific Conference Series Vol. 52, Astronomical Data Analysis Software and Systems II. p. 246

Le Bertre T., 1992, A\&AS, 94, 377

Lenz P., Breger M., 2004, in Zverko J., Ziznovsky J., Adelman S. J., Weiss W. W., eds, IAU Symposium Vol. 224, The AStar Puzzle. pp 786-790, doi:10.1017/S1743921305009750

Lenzen R., et al., 2003, in Iye M., Moorwood A. F. M., eds, Proc. SPIEVol. 4841, Instrument Design and Performance for Optical/Infrared Ground-based Telescopes. pp 944-952, doi:10.1117/12.460044

Liljegren S., Höfner S., Nowotny W., Eriksson K., 2016, A\&A, 589, A130

Lopez B., Perrier C., Mekarnia D., Lefevre J., Gay J., 1993, A\&A, 270,462

Lykou F., et al., 2015, A\&A, 576, A46

Maercker M., et al., 2012, Nature, 490, 232

Mattsson L., 2010, A\&A, 515, A68

Mattsson L., Wahlin R., Höfner S., 2010, A\&A, 509, A14

Mauron N., Huggins P. J., 2006, A\&A, 452, 257

Mayer A., et al., 2013, A\&A, 549, A69

Mayer A., et al., 2014, A\&A, 570, A113

McMullin J. P., Waters B., Schiebel D., Young W., Golap K., 2007, in Shaw R. A., Hill F., Bell D. J., eds, Astronomical Society of the Pacific Conference Series Vol. 376, Astronomical Data Analysis Software and Systems XVI. p. 127

Meadows P. J., Good A. R., Wolstencroft R. D., 1987, MNRAS, $225,43 \mathrm{P}$

Menzies J. W., Feast M. W., Whitelock P. A., 2006, MNRAS, 369,783

Miller Bertolami M. M., 2016, A\&A, 588, A25

Mohamed S., Podsiadlowski P., 2012, Baltic Astronomy, 21, 88

Monet D. G., et al., 2003, AJ, 125, 984

Monnier J. D., Tuthill P. G., Danchi W. C., 2000, ApJ, 545, 957

Monnier J. D., et al., 2014, in Optical and Infrared Interferometry IV. p. $91461 Q$, doi:10.1117/12.2057312

Mowlavi N., et al., 2018, preprint, p. arXiv:1805.02035 (arXiv:1805.02035)

Neufeld D. A., et al., 2011, ApJ, 727, L29

Neugebauer G., et al., 1984, ApJ, 278, L1

Nowotny W., Lebzelter T., Hron J., Höfner S., 2005, A\&A, 437,285

Nowotny W., Aringer B., Höfner S., Lederer M. T., 2011, A\&A, 529, A129

Nowotny W., Aringer B., Höfner S., Eriksson K., 2013, A\&A, 552, A20

Nyman L.-A., Olofsson H., Johansson L. E. B., Booth R. S., Carlstrom U., Wolstencroft R., 1993, A\&A, 269, 377 
Ohnaka K., Driebe T., Weigelt G., Wittkowski M., 2007, A\&A, 466, 1099

Ohnaka K., Hofmann K. H., Schertl D., Weigelt G., Malbet F., Massi F., Meilland A., Stee P., 2012, A\&A, 537, A53

Ohnaka K., Schertl D., Hofmann K. H., Weigelt G., 2015, A\&A, 581, A127

Olivier E. A., Wood P. R., 2003, ApJ, 584, 1035

Parker Q. A., et al., 2006, MNRAS, 373, 79

Pietrzyński G., et al., 2013, Nature, 495, 76

Price S. D., Smith B. J., Kuchar T. A., Mizuno D. R., Kraemer K. E., 2010, ApJS, 190, 203

Ramstedt S., Olofsson H., 2014, A\&A, 566, A145

Ramstedt S., et al., 2014, A\&A, 570, L14

Rau G., Paladini C., Hron J., Aringer B., Groenewegen M. A. T., Nowotny W., 2015, A\&A, 583, A106

Rau G., Hron J., Paladini C., Aringer B., Eriksson K., Marigo P., Nowotny W., Grellmann R., 2017, A\&A, 600, A92

Roeser S., Demleitner M., Schilbach E., 2010, AJ, 139, 2440

Rousset G., et al., 2003, in Wizinowich P. L., Bonaccini D., eds, Proc. SPIEVol. 4839, Adaptive Optical System Technologies II. pp 140-149, doi:10.1117/12.459332

Ryde N., Schöier F. L., Olofsson H., 1999, A\&A, 345, 841

Sanchez-Bermudez J., Hummel C. A., Tuthill P., Alberdi A., Schödel R., Lacour S., Stanke T., 2016, A\&A, 588, A117

Schlafly E. F., Finkbeiner D. P., 2011, ApJ, 737, 103

Schöier F. L., Ryde N., Olofsson H., 2002, A\&A, 391, 577

Schöier F. L., Bast J., Olofsson H., Lindqvist M., 2007, A\&A, 473,871

Schöier F. L., Ramstedt S., Olofsson H., Lindqvist M., Bieging J. H., Marvel K. B., 2013, A\&A, 550, A78

Schröder K. P., Sedlmayr E., 2001, A\&A, 366, 913

Smith C., Zijlstra A. A., Decin L., Lombaert R., 2012, in Nuclei in the Cosmos (NIC XII). p. 233

Soszyński I., Wood P. R., 2013, ApJ, 763, 103

Stewart P. N., Tuthill P. G., Monnier J. D., Ireland M. J., Hedman M. M., Nicholson P. D., Lacour S., 2016, MNRAS, 455, 3102

Thiébaut E., 2008, in Optical and Infrared Interferometry. p. 70131I, doi:10.1117/12.788822

Tuthill P. G., Monnier J. D., Danchi W. C., Wishnow E. H., Haniff C. A., 2000a, PASP, 112, 555

Tuthill P. G., Monnier J. D., Danchi W. C., Lopez B., 2000b, ApJ, 543, 284

Tuthill P., et al., 2010, in Ground-based and Airborne Instrumentation for Astronomy III. p. 77351 O (arXiv:1006.2586), doi:10.1117/12.856806

Westerlund B. E., Olander N., 1978, A\&AS, 32, 401

Whitelock P. A., Feast M. W., Marang F., Groenewegen M. A. T., 2006, MNRAS, 369, 751

Wittkowski M., et al., 2017, A\&A, 601, A3

Wittkowski M., et al., 2018, preprint, p. arXiv:1805.05333 (arXiv: 1805.05333)

Wood P. R., Nicholls C. P., 2009, ApJ, 707, 573

Woods P. M., Schöier F. L., Nyman L.-A., Olofsson H., 2003, A\&A, 402, 617

Zacharias N., Monet D. G., Levine S. E., Urban S. E., Gaume R., Wycoff G. L., 2004, in American Astronomical Society Meeting Abstracts. p. 1418

van Leeuwen F., Feast M. W., Whitelock P. A., Yudin B., 1997, MNRAS, 287, 955

This paper has been typeset from a $\mathrm{TE}_{\mathrm{E}} \mathrm{X} / \mathrm{LATEX}$ file prepared by the author. 